\title{
Ergebnisse der Umfrage: «Status quo: Kundennutzen durch digitale Transformation?»
}

Denisa Kykalová, Elke Brucker-Kley und Simon Näpflin

2.1 Strategische Ausrichtung - 24

2.2 Prozessdigitalisierung - 27

2.3 Digitale Transformation - 31

2.4 Was will der Kunde? - 33

2.5 Kundenperspektive in der Prozessgestaltung und -optimierung - 36

2.6 Kundendaten: Quellen, Nutzung und Einsatz für die Prozessausführung - 41 
Welchen Beitrag leistet das Prozessmanagement für die Maximierung des Kundennutzens? Wie lassen Unternehmen die Kundenperspektive in die Prozessgestaltung einfliessen? In welcher Ausprägung setzen Unternehmen Kunden- und Prozessdaten ein, um Kundenerlebnisse zu individualisieren? Ist dabei Transparenz und Datenherrschaft für Kunden sichergestellt? Erheben Unternehmen systematisch Digitalisierungspotenzial in ihren Prozessen? Wie steht es um die Durchgängigkeit der Prozesse und welche Formen der Prozessdigitalisierung kommen dabei zum Einsatz? Diese und weitere Fragen standen im Mittelpunkt der Online-Befragung, die im Rahmen der vorliegenden Studie im Mai 2016 durchgeführt wurde.

«Welche Rolle spielt das Prozessmanagement als Gestaltungselement der digitalen Transformation?» lautet die Forschungsfrage der BPM-Studie 2016, die sich den Themenschnittstellen der drei Wirkungsfelder Kundenutzen - Digitalisierung - Prozessmanagement widmet. Ziel der quantitativen Befragung war es, den Status quo des Methoden- und Werkzeugeinsatzes innerhalb und an den Schnittstellen dieser drei Wirkungsfelder zu erheben und Erkenntnisse über die Ausrichtung und den Reifegrad des Prozessmanagements in Unternehmen im Hinblick auf die Herausforderungen des technologischen Wandels zu gewinnen. Ausgehend von den in Abschn. 1.2 formulierten Forschungsfragen und Hypothesen sowie dem in Abschn. 1.3 vorgestellten Rahmenwerk wurden für die quantitative Erhebung Unterforschungsfragen in sechs Themenbereichen formuliert (- Abb. 2.1) und ein Fragenkatalog ausgearbeitet ${ }^{1}$. Die Ergebnisse der Online-Befragung werden in den nachfolgenden Kapiteln nach diesen sechs Themenbereichen gegliedert präsentiert.

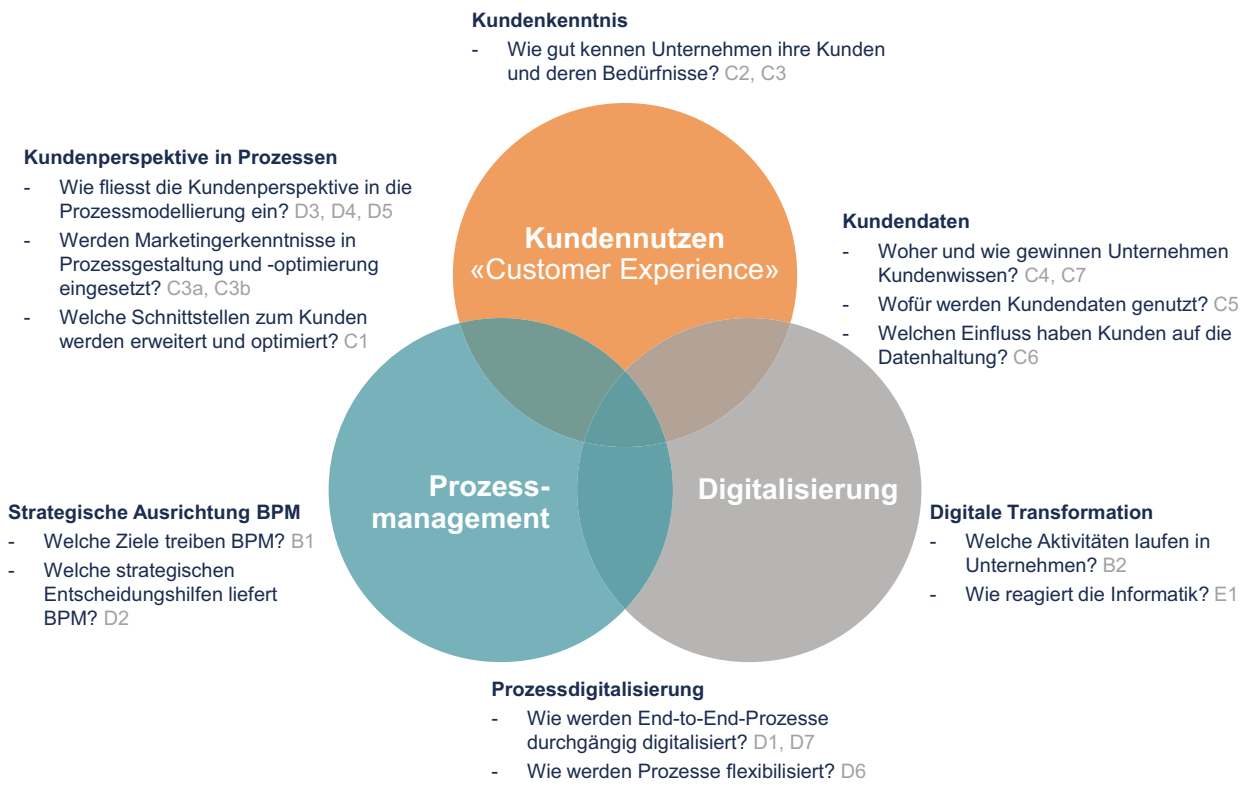

- Abb. 2.1 Unterforschungsfragen für die 6 Themenbereiche

\footnotetext{
${ }^{1}$ Der vollständige Fragenkatalog findet sich im Anhang der Studie. Das Studiendesign sowie die zugrundeliegenden Forschungsfragen und Hypothesen sind Gegenstand von Abschn. 1.2.
} 
- Abb. 2.2 Teilnehmende nach Grösse des Unternehmens (KMU vs. Grosse Unternehmen)

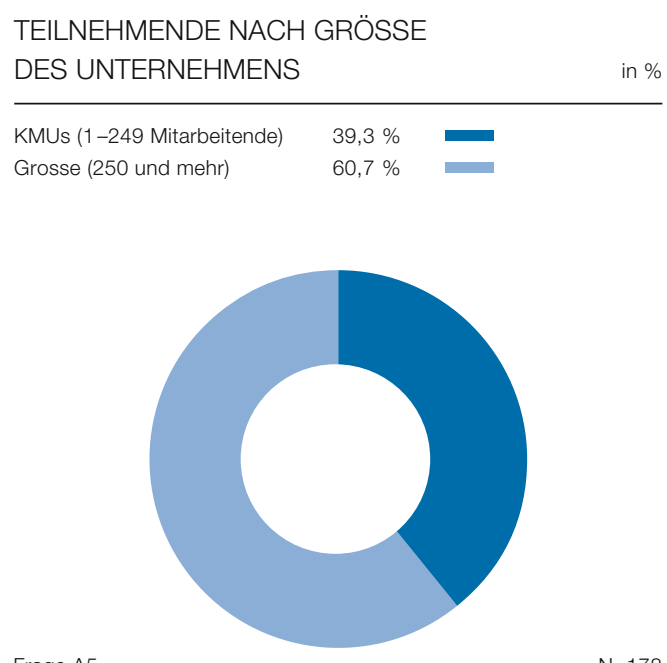

Frage A5 $N=178$

Die Online-Befragung richtete sich an Unternehmen im deutschsprachigen Raum und wurde mehrheitlich von Teilnehmenden aus Unternehmen mit Tätigkeitsschwerpunkt in der Schweiz (81\%) beantwortet ${ }^{2}$ (- Abb. 2.2). 178 Personen aus unterschiedlichen Funktionsbereichen und aus Unternehmen diverser Branchen haben sich an der Online-Umfrage beteiligt und haben dazu beigetragen, den Status quo des Prozessmanagements im Kontext der digitalen Transformation zu erheben. Fast drei Viertel der Befragten sind in der Unternehmensleitung (20\%), im Projekt-, Qualitäts- oder Prozessmanagement oder in der Informatik tätig und stellen eine unternehmensweite Betrachtung der erhobenen Themenkreise sicher. Nahezu 80 \% der Befragten nehmen eine spezifische Prozessmanagement-Funktion (z. B. Prozessverantwortlicher, Process-Manager, Chief Process Officer etc.) wahr, so dass von einem fundierten Fach- und Erfahrungswissen bei der Beantwortung der Fragen ausgegangen werden kann.

Der Blick auf die Unternehmensgrösse der Teilnehmenden zeigt ein Verhältnis von 1:1,5 zwischen KMUs und grossen Unternehmen: 70 befragte Personen repräsentieren dabei kleinste, kleine und mittlere Unternehmen (KMU), was 39,3\% der Gesamtteilnehmerzahl entspricht, 108 Befragte bzw. 60,7\% gehören grossen Unternehmen (in den folgenden Abbildungen als GrossU bezeichnet) mit 250 oder mehr Mitarbeitenden $\mathrm{an}^{3}$.

\footnotetext{
${ }^{2}$ Die vollständigen Daten zur Zusammensetzung des Teilnehmerkreises der Online-Befragung finden sich im Anhang der Studie.

${ }^{3}$ In einigen der folgenden Auswertungen wird auf die relative Beteiligung der Unternehmen zu ihrer Grössen-Gruppe, die gesamthaft an der Umfrage teilgenommen hat, hingewiesen (z. B. «29\% der befragten KMUs und $42 \%$ der befragten grossen Unternehmen geben Transparenz als wichtigsten strategischen Treiber für das Prozessmanagement an», vgl. - Abb.2.5). Dies soll helfen, grössentypische Fokus-, Reifegrad- und Vorgehensunterschiede zu erkennen. Wird auf die Unternehmensgrösse nicht eingegangen, gibt es keine signifikanten Unterschiede zwischen den Grössengruppen.
} 


\subsection{Strategische Ausrichtung}

Transparenz und Wirtschaftlichkeit sind die etablierten Zielsetzungen des strategischen Prozessmanagements in Unternehmen. Die Studie geht von der Hypothese aus, dass Prozessmanagement auch einen relevanten Beitrag leisten kann, um Kundenerlebnisse zu optimieren oder Produkte, Dienstleistungen und Geschäftsmodelle zu innovieren. Um dies zu erreichen, müssen die Weichen bereits bei der strategischen Ausrichtung des Prozessmanagements gestellt werden. Haben Organisationen die Weichen entsprechend gestellt?

Die Frage nach den drei wichtigsten strategischen Treibern für das Prozessmanagement ergibt ein gewohntes Bild (- Abb. 2.3): Wie bereits in der letztjährigen BPM-Studie (BruckerKley, et al., 2015) ist die Steigerung der Wirtschaftlichkeit die Topmotivation für das Prozessmanagement (83\%), dicht gefolgt von den Transparenzthemen (78 \%) Prozessbewusstsein, Qualitätssicherung und Governance/Risk/Compliance (GRC). An Relevanz gewonnen hat erneut die Kundenzufriedenheit (64\%), die 2015 bereits $49 \%$ der Befragten als Motivation angegeben hatten. Treiber wie Flexibilität (34\%) und Innovation (25\%) hingegen, die im Kontext der digitalen Transformation eine zentrale Rolle spielen, stehen für Unternehmen weniger im Mittelpunkt des Prozessmanagements, nahmen gegenüber dem Vorjahr jedoch um $10 \% \mathrm{zu}$. Der Blick auf die Unternehmensgrösse zeigt (ohne Abbildung), dass die KMUs, relativ betrachtet, häufiger als die grossen Unternehmen Kundenzufriedenheit ( $71 \%$ aller befragten KMUs, $63 \%$ aller befragten GrossU) und Innovation (34 \% der KMUs, $21 \%$ der GrossU) als Motivationen für das Prozessmanagement nennen. Diese Themen scheinen für KMUs wichtiger zu sein als für grosse Unternehmen. Dies bestätigen auch die weiteren Befragungsergebnisse (vgl.

- Abb. 2.7 und 2.24).

Ein interessantes Bild ergibt die Frage nach der Priorisierung der drei genannten Ziele von 1 (höchste Priorität) bis 3 (• Abb. 2.4). Hier zeigt sich, dass Transparenz immer noch die vorrangigste Motivation für das Prozessmanagement der befragten Unternehmen ist (36\%), gefolgt von Kundenzufriedenheit (28\%) und dem auf den ersten Blick dominierenden Wirtschaftlichkeitsthema (26\%). Dieses Resultat unterstreicht die hohe Relevanz der Transparenz als wesentliche Voraussetzung für die nachgelagerte Operationalisierung, Standardisierung und Optimierung der Prozesse.

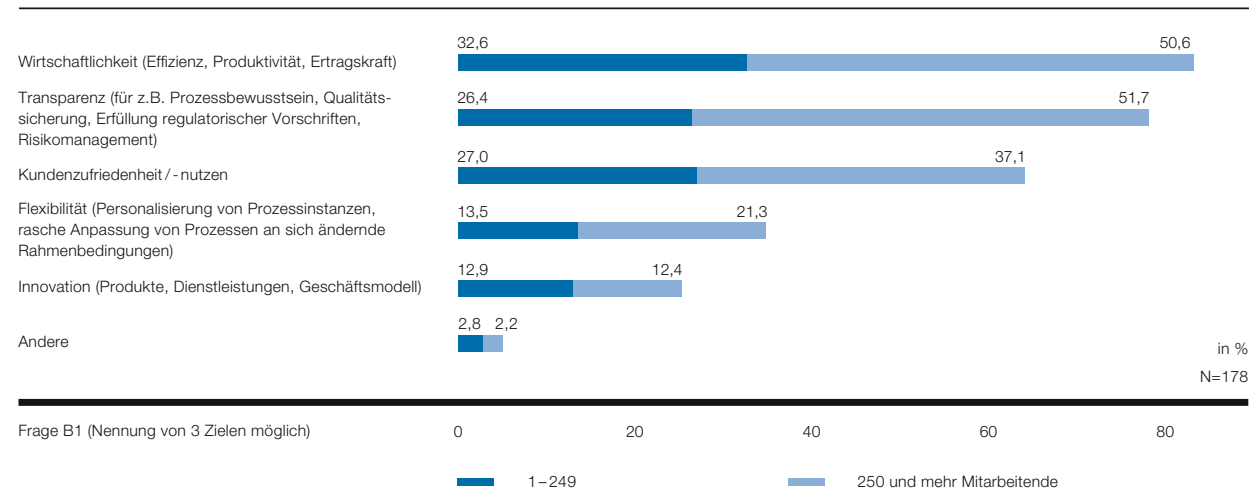

- Abb. 2.3 Strategische Ziele des Prozessmanagements 
- Abb. 2.4 Strategische Ziele des Prozessmanagements - priorisiert

STRATEGISCHE ZIELE DES PROZESS-

MANAGEMENTS - PRIORISIERT

in $\%$

$\begin{array}{lll}\text { Wirtschaftlichkeit } & \text { Flexibilität } \\ \text { Transparenz } & \text { Innovation } \\ \text { Kundenzufriedenheit/-nutzen } & \text { Andere }\end{array}$

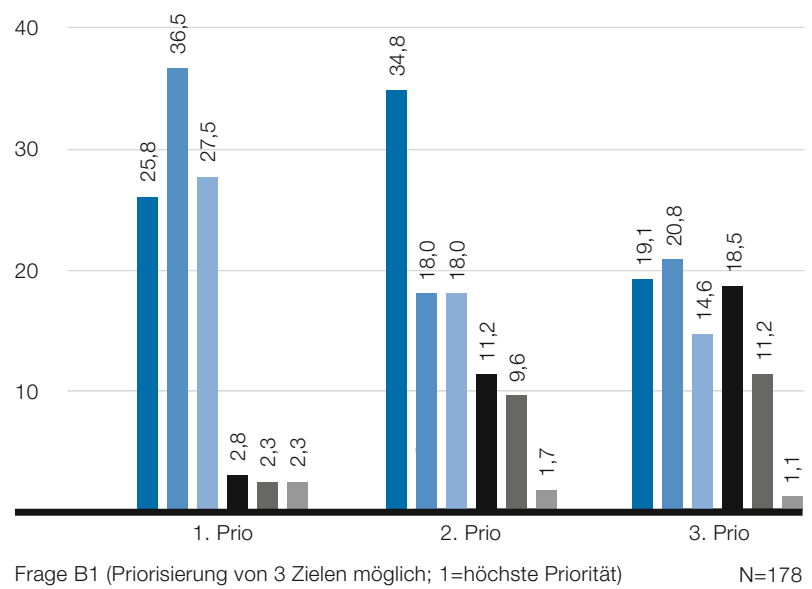

Der Detailblick auf die Ziele erster Priorität nach Unternehmensgrösse (• Abb. 2.5) zeigt, dass bei den KMUs Transparenz, Kundenzufriedenheit und Wirtschaftlichkeit ähnlich hoch gewichtet wurden. Bei den grossen Unternehmen dominiert klar die Transparenz mit $42 \%$, gefolgt von Kundenzufriedenheit (28\%) und Wirtschaftlichkeit (25\%). Interessant ist, dass Innovation nur von KMUs als wichtigster BPM-Treiber gewählt wurde. Die absolute Zahl ist jedoch zu klein (4 Teilnehmende), um Schlussfolgerungen ziehen zu können.

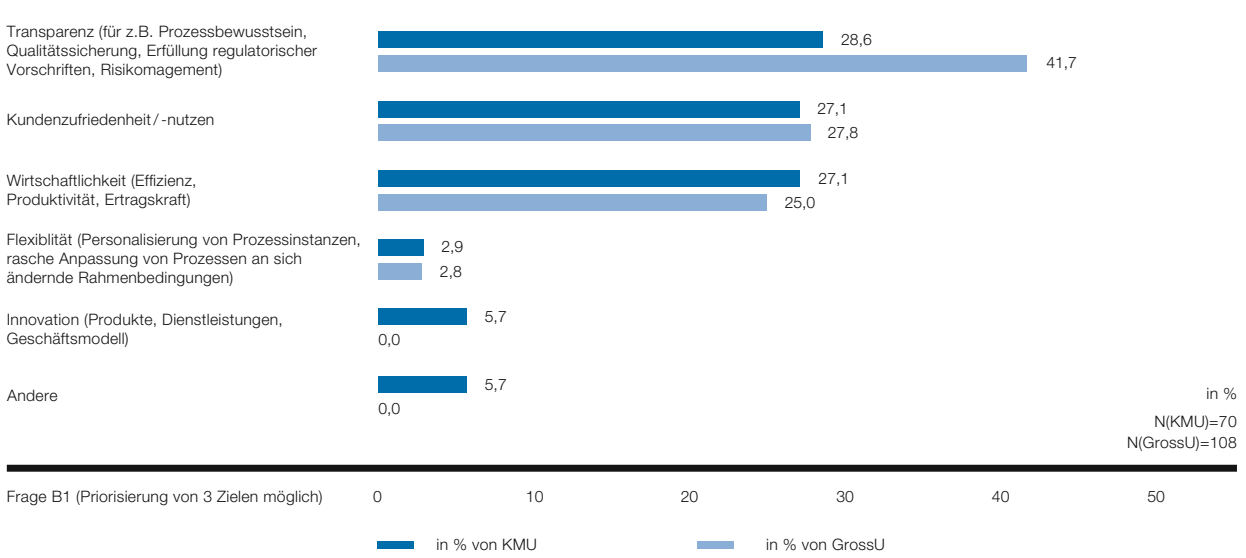

- Abb. 2.5 Strategische Ziele des Prozessmanagements - «1. Priorität» nach Unternehmensgrösse 
- Strategische Impulse durch Potenzialanalysen

Unternehmen sehen sich im Wettbewerb mit einem hohen Druck konfrontiert, auf den digitalen Wandel zu reagieren. Offen bleibt die Frage, wo sie mit der Digitalisierung beginnen sollen, das heisst, wie sie Anwendungsfelder und Technologien mit einem hohen Nutzenpotenzial identifizieren können. Die Studie geht von der Hypothese aus, dass Unternehmen das Prozessmanagement noch nicht systematisch einsetzen, um Digitalisierungspotenziale zu erkennen, zum Beispiel indem sie relevante Informationen in strategischen Prozesslandkarten oder Prozessmodellen visualisieren bzw. hinterlegen oder operative Prozessdaten analysieren.

Die Frage nach diesbezüglichen Aktivitäten führt jedoch zu einem optimistischeren Bild (- Abb. 2.6): Zwei Drittel der Unternehmen führen gelegentlich oder systematisch Potenzialanalysen in mindestens einem der erfragten Bereiche durch. Mehr als drei Viertel der Unternehmen identifizieren erfolgskritische Prozesse oder Prozesse, die für die Kundenzufriedenheit kritisch sind. Ähnlich viele Unternehmen identifizieren Standardisierungs- und Automatisierungspotenzial sowie Digitalisierungspotenziale für kollaborative, schwach strukturierte Prozesse oder Kundeninteraktionen. Ernüchternd hingegen ist der geringe Anteil der Unternehmen, die diese Potenzialanalysen systematisch durchführen, was Zweifel an der Wirkungskraft der durchgeführten Analysen aufkommen lässt. Insbesondere Analysen, die Digitalisierungspotenziale über die Unternehmensgrenzen hinweg (17\%) sowie Sourcing-/Outsourcingpotenziale $(11 \%)$ ermitteln, werden kaum systematisch durchgeführt. Auch die für das Kundenerlebnis wichtigen Optimierungspotenziale von Nutzeroberflächen (UX) werden noch kaum systematisch identifiziert (13\%).

Die Detailauswertung nach Unternehmensgrösse (- Abb. 2.7) offenbart, dass beide Gruppen ähnlich aktiv sind und zumindest gelegentlich Potenzialanalysen durchführen (zwischen 54 und $89 \%$ der jeweiligen Grössengruppe). Bei der Identifizierung der kundenkritischen Prozesse sind jedoch die KMUs leicht aktiver ( $89 \%$ vs. $82 \%$ von grossen Unternehmen), für die diese Analyse die meist eingesetzte ist. Bei den grossen Unternehmen liegt ein etwas stärker ausgeprägter Fokus auf der Erhebung des Standardisierungs- und Automatisierungspotenzials

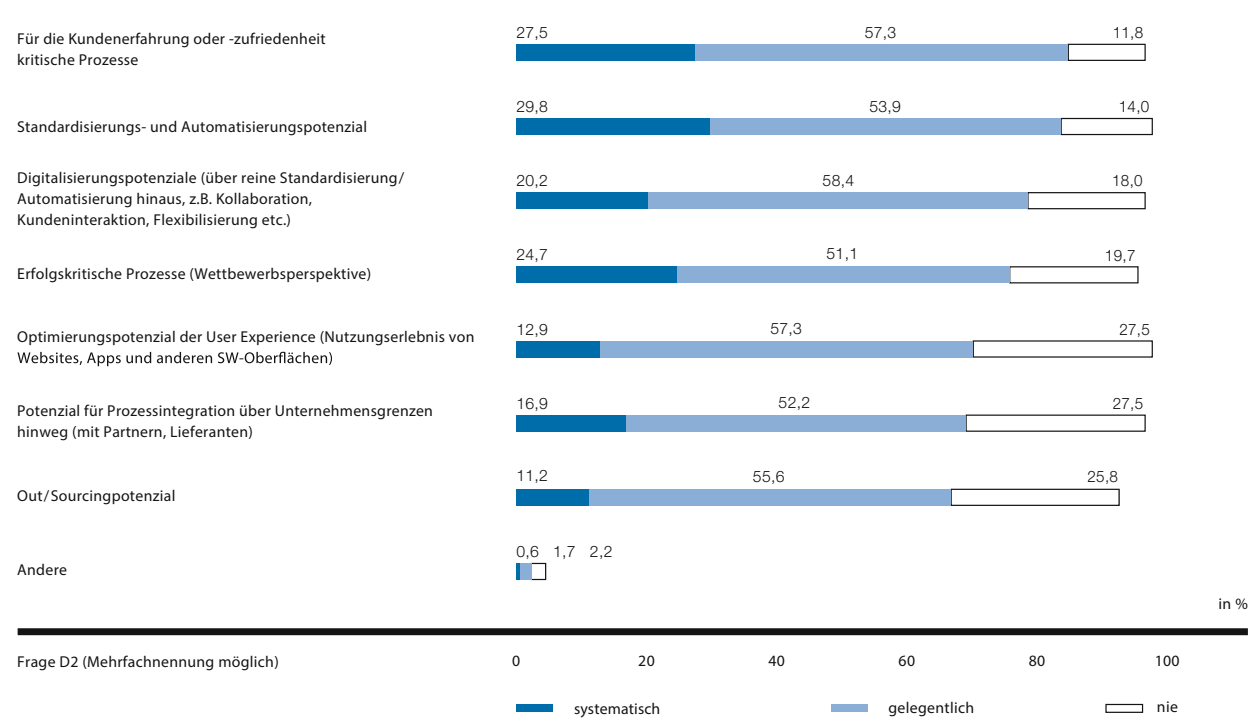

D Abb. 2.6 Potenzialanalysen für die Unterstützung strategischer Entscheide 


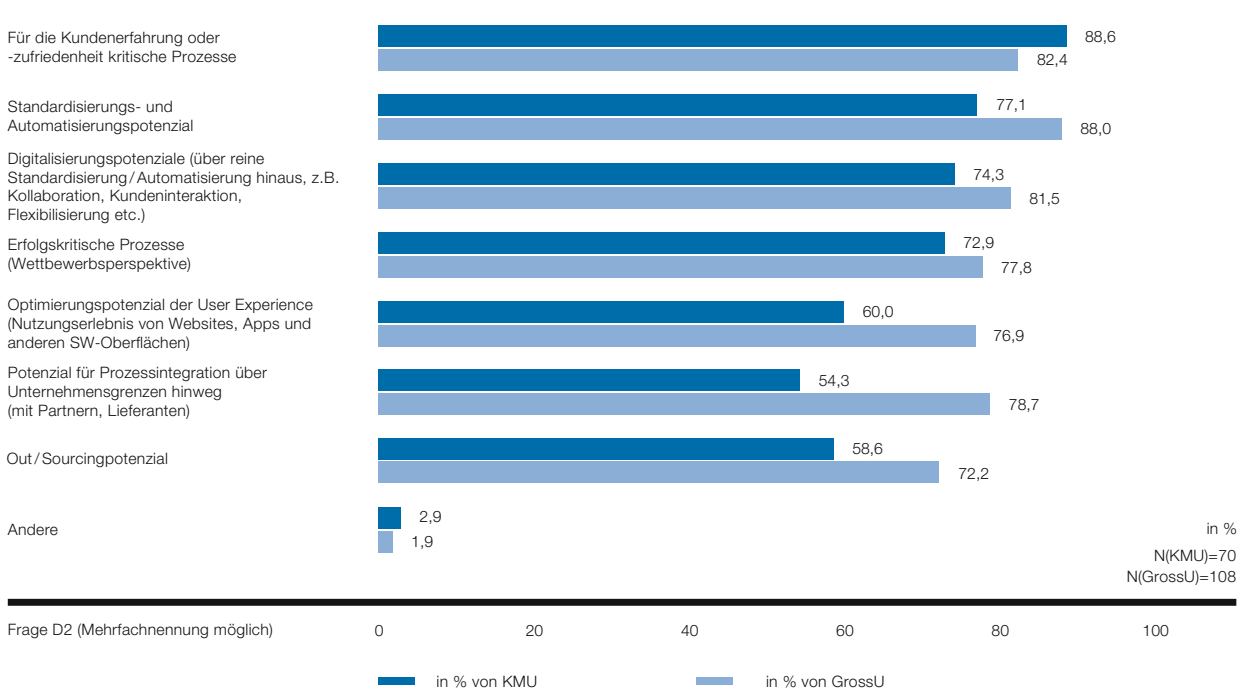

- Abb. 2.7 Prozessmanagement-Mittel für strategische Entscheidungsunterstützung - «systematisch \& gelegentlich» nach Unternehmensgrösse

vor. Dieses Resultat entspricht den oben identifizierten Zielprioritäten nach Unternehmensgrösse.

\subsection{Prozessdigitalisierung}

Modellierung, Standardisierung und Automatisierung sind die klassischen Methoden des Prozessmanagements, um Transparenz und Wirtschaftlichkeitsziele zu erreichen. Prozesse durchgängig zu machen und Medienbrüche zu beseitigen, sind die zentralen Bestrebungen. Sollen jedoch Prozesse über die Unternehmensgrenzen hinaus in Richtung Kunden oder Partner geöffnet werden und sich gar flexibel an den Kontext des Kunden anpassen, muss das Standardrepertoire der Prozessdigitalisierung erweitert werden.

\section{- Durchgängigkeit der Prozesse}

Durchgängige Prozesse sind eine wesentliche Voraussetzung sowohl für Effizienz als auch für bessere Kundenerlebnisse. Bearbeitungs- und Reaktionszeiten werden verkürzt. Kundenanliegen können rasch und im Idealfall in Echtzeit erledigt werden. Der hohe Stellenwert der Wirtschaftlichkeit und der Kundenzufriedenheit als Ziel des Prozessmanagements (• Abb. 2.3) und die Tatsache, dass über $80 \%$ der befragten Organisationen Standardisierungs- und Automatisierungspotenziale erheben, könnte den Schluss nahelegen, dass Unternehmen mit Zielrichtung «Operational und Service Excellence» in die Durchgängigkeit ihrer Prozesse investieren. Ein Blick in den Alltag der befragten Unternehmen zeigt jedoch, dass Scannen, Drucken und das handschriftliche Unterzeichnen von Dokumenten bei der Hälfte der befragten Unternehmen noch zum Unternehmensalltag gehören (• Abb. 2.8). Die restlichen Unternehmen geben an, dass dieser Umgang mit Papierdokumenten nicht mehr täglich geschieht. Nur ein sehr geringer Anteil ist in der Digitalisierung so weit fortgeschritten, dass Papier komplett aus der Aufgabenerledigung verbannt ist ( $<9 \%$ beim Drucken, $<6 \%$ beim Scannen, $<5 \%$ beim 


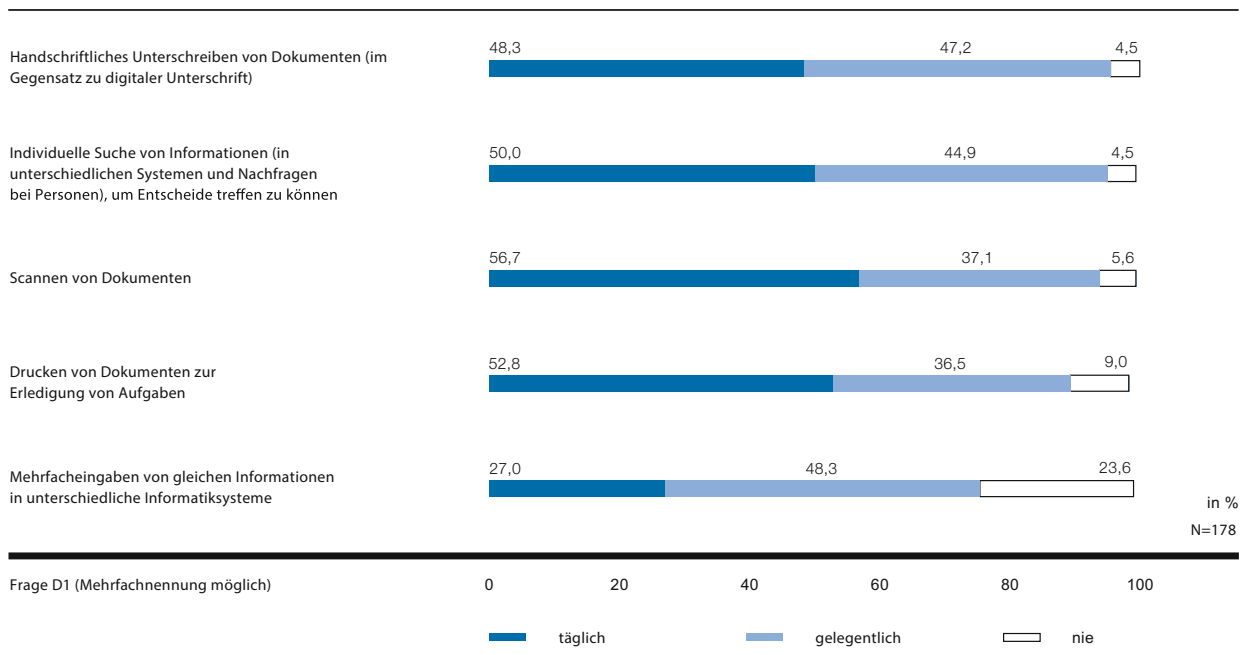

- Abb. 2.8 Medienbrüche im Unternehmensalltag

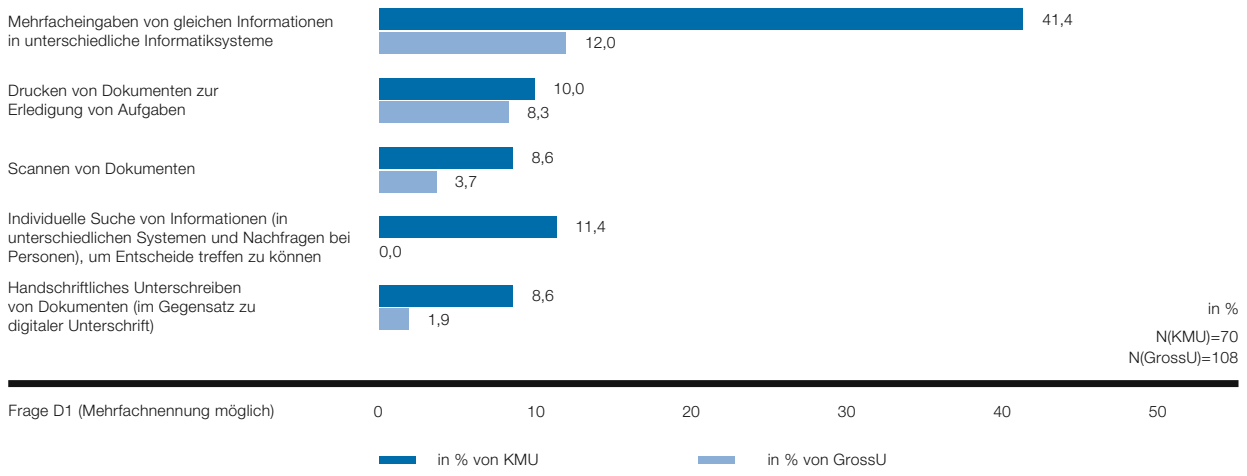

D Abb. 2.9 Medienbrüche im Unternehmensalltag - «Nie» nach Unternehmensgrösse

Unterschreiben). Auch bei der Systemintegration sind Defizite vorhanden: Bei rund der Hälfte der Unternehmen gehört die Suche in verschiedenen isolierten Informationssystemen, um Entscheide treffen zu können, zum Alltag (50 \%). In weiteren $45 \%$ ist dies gelegentlich erforderlich. Mehrfacheingaben von gleichen Informationen in unterschiedliche Informationssysteme hingegen konnten in immerhin $23 \%$ der befragten Unternehmen bereits eliminiert und in weiteren $48 \%$ reduziert werden, sind aber in mehr als einem Viertel der Unternehmen immer noch alltäglich (27\%).

Interessant ist, dass verhältnismässig mehr KMUs angeben, dass diese Tätigkeiten bei ihnen nie vorkommen (-Abb. 2.9), was auf eine höhere Durchgängigkeit der Prozesse, das heisst weniger Medienbrüche und bessere Systemintegration, hindeutet. Insbesondere bei den Mehrfacheingaben von gleichen Informationen in unterschiedlichen Systemen scheint die Grösse des Unternehmens eine Rolle zu spielen: 41 \% der KMUs geben an, nie Mehrfacheingaben tätigen zu müssen, bei grossen Unternehmen sind es nur $12 \%$. Eine Erklärung könnte darin liegen, 


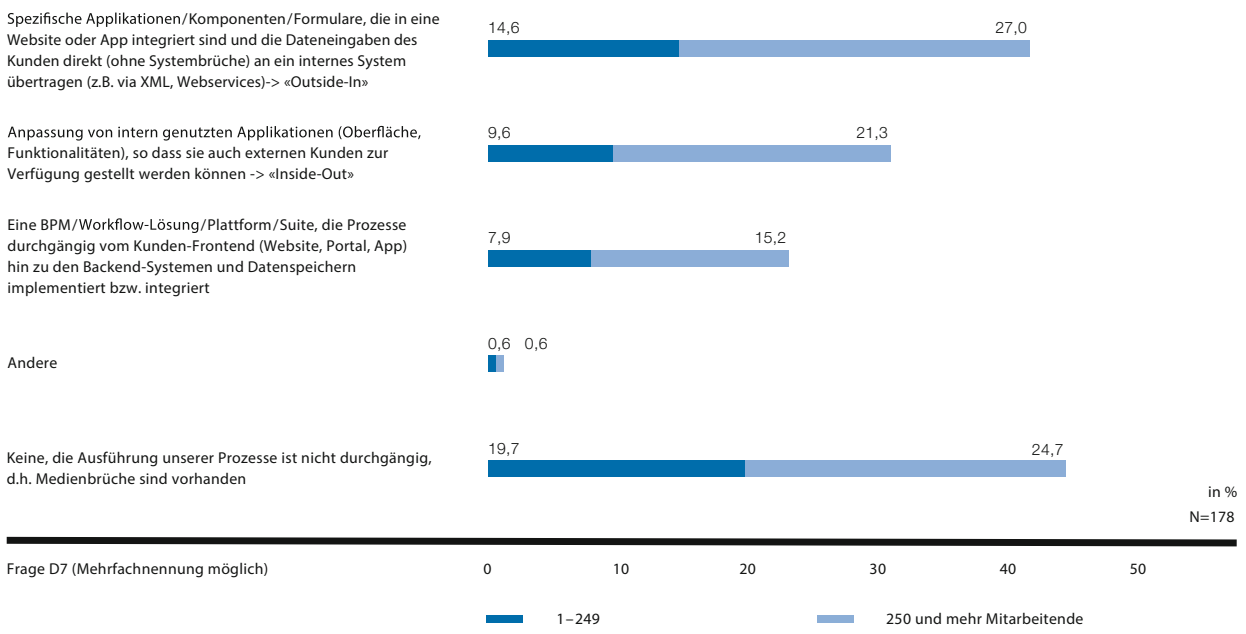

- Abb. 2.10 Einsatz technischer Methoden für durchgängige Prozesse vom und zum Kunden

dass die IT-Landschaften der KMUs weniger komplex als bei grossen Unternehmen sind und deshalb die Integration der Systeme und die Durchgängigkeit der Prozesse einfacher umsetzbar ist.

Die mediale Präsenz des digitalen Wandels ist stark von Optimierungen und Erweiterungen im Front-End in Form von mobilen Applikationen, sozialen Plattformen etc. geprägt. Dies garantiert jedoch noch keine Durchgängigkeit der Prozesse Front-to-Back bzw. Back-toFront. Die Frage nach den Methoden, die Unternehmen einsetzen, um Prozesse durchgängig von und zum Kunden-Front-End zu realisieren, offenbart den Stand dieser Durchgängigkeit (- Abb. 2.10): 44 \% der befragten Unternehmen räumen ein, dass sie keine Methoden einsetzen, und ihre Prozesse folglich noch nicht durchgängig sind und Medienbrüche aufweisen. Wenn Durchgängigkeit realisiert wird, dann implementieren Unternehmen diese mehrheitlich ausgehend vom Front-End, indem sie Dateneingaben des Kunden z. B. über Web Services an interne Systeme übertragen (41\%). Die zweithäufigste Strategie ist die Simplifizierung oder Optimierung von internen Applikationen, so dass auch Kunden oder Partner diese Systeme und Oberflächen nutzen können (31\%). Einen plattformbasierten Ansatz über eine BPModer Workflow-Lösung wählen hingegen nur $23 \%$ der befragten Unternehmen. Dies könnte darauf hinweisen, dass Unternehmen die durchgängige Digitalisierung von Prozessen projektbezogen, ausgehend von individuellen Anwendungsfällen und Business Cases, aber nicht unternehmensweit angehen.

Unternehmen scheinen diese Defizite der Prozessdurchgängigkeit erkannt zu haben und investieren deshalb aktuell intensiv in Automatisierung bzw. Digitalisierung durchgängiger Prozesse und den digitalen Arbeitsplatz (siehe Abschn. 2.3 Digitale Transformation, - Abb. 2.12).

\section{- Flexibilisierung der Prozesse}

Durchgängige Prozesse sind ein erster Schritt, um Kundenerlebnisse und den digitalen Arbeitsplatz im Unternehmen zu optimieren. Bestimmte Anwendungsszenarien oder Zielgruppen 


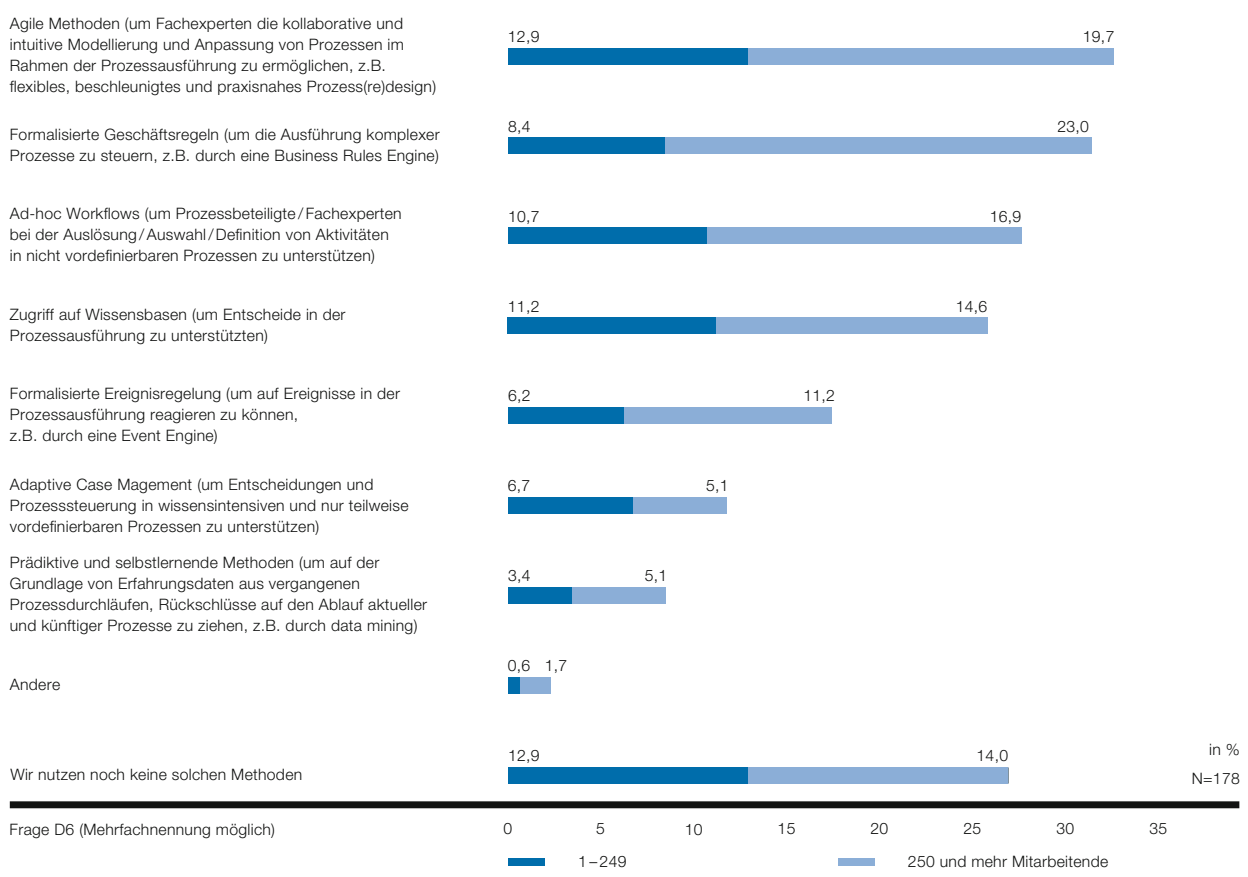

- Abb. 2.11 Einsatz von Methoden für flexible und kontextsensitive Prozesse

verlangen jedoch nach mehr. Prozesse sollen flexibel bzw. individualisiert ablaufen, indem sie sich in (Fast-) Echtzeit auf den aktuellen Kontext, die Eigenschaften oder das Verhalten eines Kunden ausrichten. Mitarbeitende sollen durch eine flexible und informationsreiche IT-Unterstützung in wissensintensiven und schwach strukturierten Prozessen befähigt werden. Die Frage nach dem Einsatz solcher Methoden und Werkzeuge ergibt ein durchwachsenes Bild (- Abb. 2.11). Mehr als ein Viertel der befragten Unternehmen gibt an, keine solchen Methoden oder Werkzeuge einzusetzen. Am stärksten verbreitet ist die Anwendung agiler Methoden für die Prozessmodellierung ( 33 \%). Im Rahmen der Prozessausführung ist, wie bereits in der letztjährigen BPM-Studie (Brucker-Kley, et al., 2015) erkennbar, der Einsatz von Business Rules am gebräuchlichsten (31 \%). Business Rules flexibilisieren Prozesse, indem die Geschäftsregeln getrennt von den eigentlichen Prozessmodellen verwaltet, wiederverwendet und angepasst werden, so dass Änderungen im Prozess rascher umsetzbar sind. Beim Einsatz von Business Rules sind grosse Unternehmen fast doppelt so aktiv wie KMUs (38 \% der GrossU vs. $21 \%$ der KMUs). Für sie scheint sich der Aufwand für die Formalisierung und Implementierung der Geschäftsregeln mehr zu rechnen als für kleinere und mittlere Unternehmen. Ad-hoc Workflows, die Prozessbeteiligte in schwach strukturierten Prozessen dabei unterstützen, Aktivitäten situationsabhängig flexibel auszulösen, auszuwählen oder zu definieren, sind auf dem Vormarsch (nur $13 \%$ in der BPM-Studie 2015 vs. 28 \% 2016). Adaptive Case Management hingegen fristet weiterhin ein Nischendasein ( $5 \%$ 2015, $12 \%$ 2016) ebenso wie andere wissensbasierte, selbstlernende Verfahren. 


\subsection{Digitale Transformation}

Digitale Transformation ist ein Hype-Thema, doch wo stehen Unternehmen, wenn es darum geht, den digitalen Wandel konkret zu implementieren? Entwickeln sie innovative digitale Produkte und Dienstleistungen? Optimieren oder erweitern sie ihre digitalen Schnittstellen zum Kunden? Bleibt das Back-End isoliert oder werden Prozesse durchgängig digitalisiert? Profitieren auch die Mitarbeitenden von innovativeren digitalen Arbeitsplätzen? Und mit welchen Mitteln reagiert die Informatik auf diese Herausforderungen?

Nomen est omen: Digitale Transformation ist kein Zustand, sondern bedeutet Veränderung. Folglich sind die befragten Unternehmen in allen erfragten Handlungsfeldern der digitalen Transformation sehr aktiv, aber vieles befindet sich noch in der Umsetzung oder wird erprobt (-Abb. 2.12). Im Unterschied zur medialen Wahrnehmung stehen dabei jedoch nicht die reinen Front-End-Themen an erster Stelle. Das Thema Automatisierung und Digitalisierung durchgängiger Prozesse führt das Feld knapp an, wobei sich Unternehmen in diesem Handlungsfeld mehrheitlich noch in der Umsetzungs- und Experimentierphase befinden. Dies korreliert mit den festgestellten Durchgängigkeitsdefiziten der Prozesse, die bei vielen Unternehmen den Alltag aktuell noch prägen (vgl. Abschn. 2.2). Ähnlich aktiv, mit einem leicht höheren Anteil bereits realisierter Projekte sind Unternehmen bei der Einführung innovativer digitaler Arbeitsplätze für ihre Mitarbeitenden. Ebenfalls mehr als $80 \%$ arbeiten an der

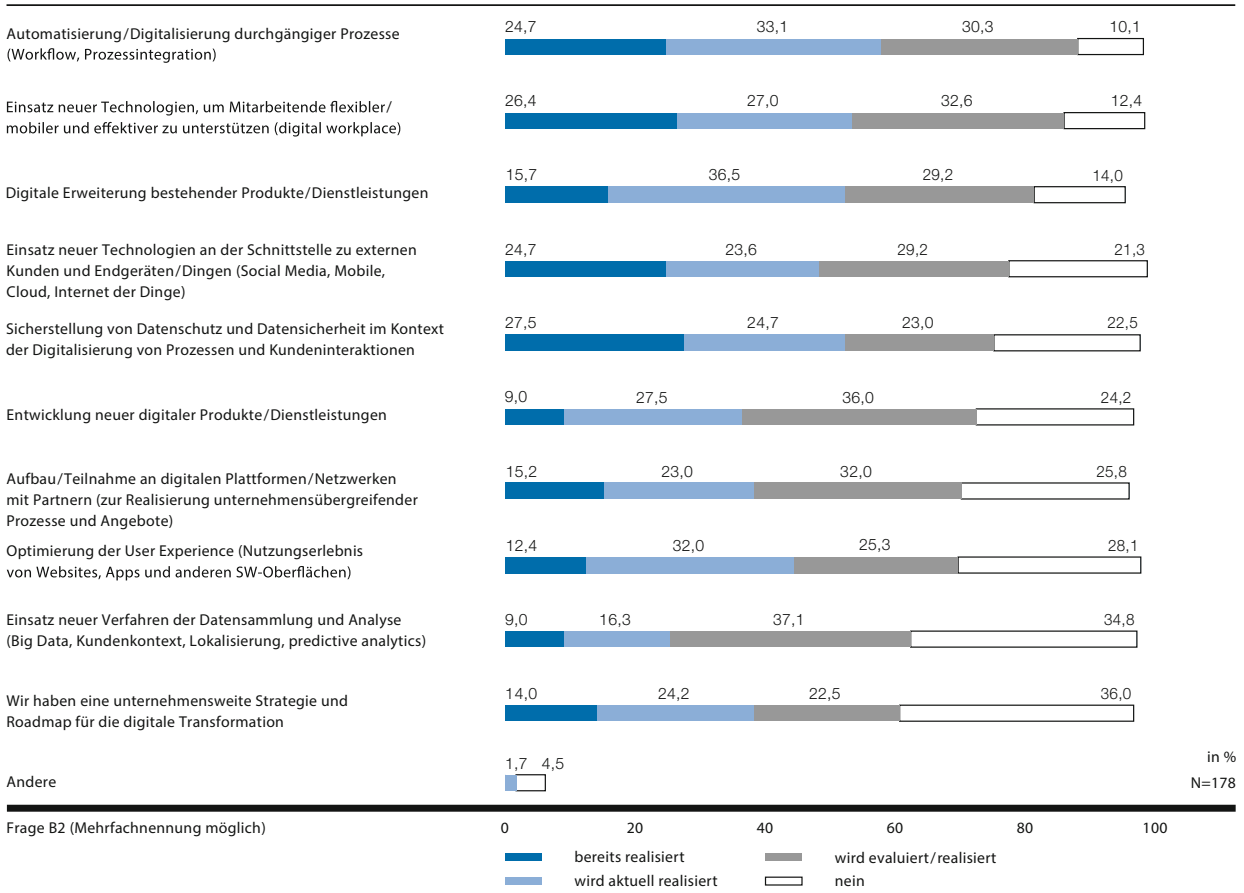

- Abb. 2.12 Aktionsfelder der digitalen Transformation 
digitalen Erweiterung bestehender Produkte oder Dienstleistungen, wobei der digitale Wandel in diesem Bereich im Vergleich zur Automatisierung und zum digitalen Arbeitsplatz noch deutlicher im Werden und Experimentieren ist. Erstaunlich ist die Tatsache, dass der Einsatz neuer Technologien an der Schnittstelle zum Kunden (Social Media, Mobile, IoT etc.) zwar im Vorderfeld liegt, aber keine Spitzenposition einnimmt (77\%). Andere wesentliche Handlungsfelder, wie die Optimierung der User Experience (vorrangig auf externe Kunden mit 67 \%, weniger auf die eigenen Mitarbeitenden mit $43 \%$, seltener auf Partner/Kooperationen mit $25 \%$ ausgerichtet ${ }^{4}$ ) und Big Data liegen zwar etwas zurück und befinden sich noch in der Umsetzung oder Experimentierphase, sind jedoch mit über $60 \%$ ebenfalls sehr präsent in den befragten Unternehmen. Ebenfalls bemerkenswert ist, dass immerhin $60 \%$ der Unternehmen eine digitale Transformationsstrategie haben oder gerade erarbeiten oder eine solche evaluieren. Verabschiedet und in Aktion ist eine solche jedoch erst bei rund $14 \%$ der befragten Unternehmen.

Die Detailauswertung nach Unternehmensgrösse (- Abb. 2.13) zeigt, dass der digitale Arbeitsplatz von KMUs und grossen Unternehmen gleichermassen angestrebt wird. Bei allen weiteren Aktionsfeldern haben die grossen Unternehmen einen Vorsprung. Insbesondere bei technologie- und kostenintensiven Aktionsfeldern wie beim Einsatz neuer Verfahren der Datensammlung und Analyse, beim Einsatz von Web-2.0-Technologien oder bei der Optimierung der User Experience fallen die Unterschiede auf. Ebenfalls leicht im Rückstand sind die KMUs

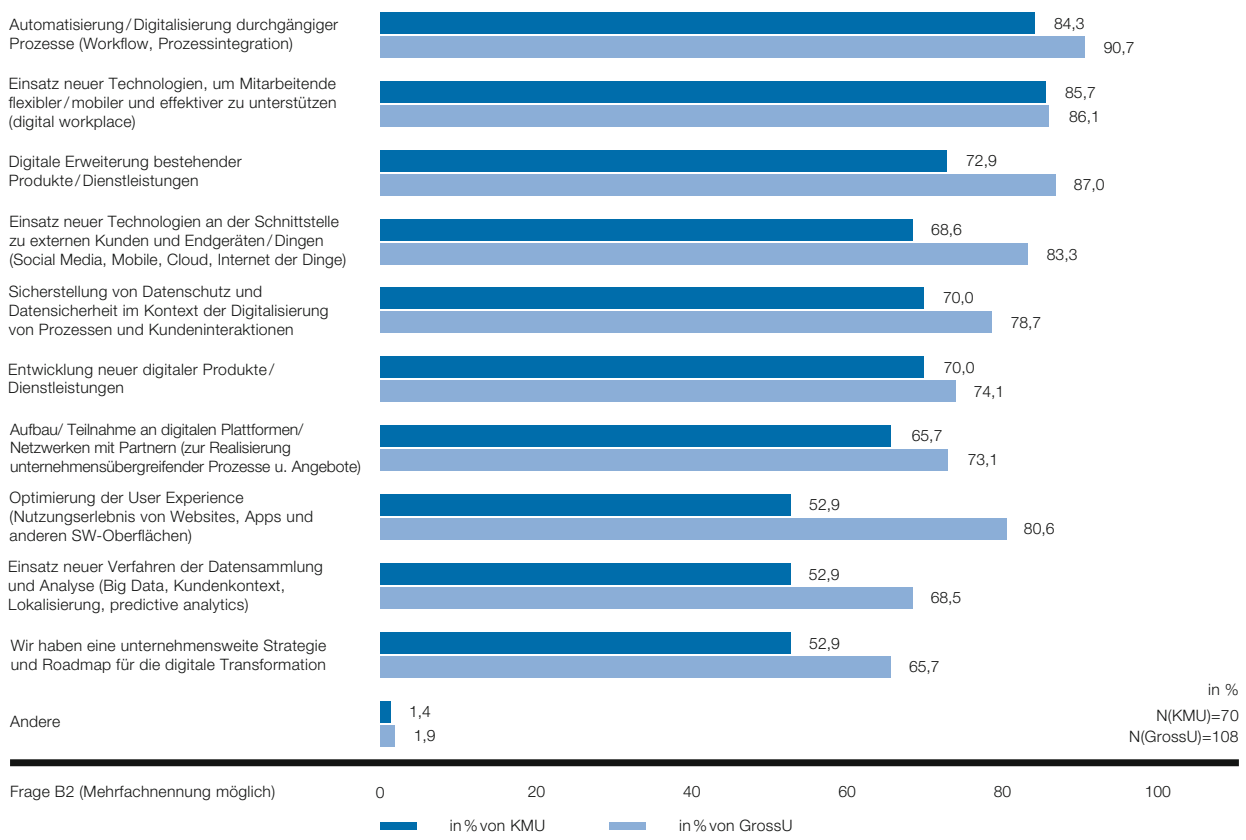

- Abb. 2.13 Aktionsfelder der digitalen Transformation - «umgesetzt oder angestrebt oder evaluiert» nach Unternehmensgrösse

\footnotetext{
${ }^{4}$ Ohne Abbildung, Unterfrage D2a nach dem Fokus bei der Optimierung der User Experience: externe Kunden, Mitarbeitende oder Partner (siehe Fragenkatalog in $>$ Abschn. 10.2).
} 


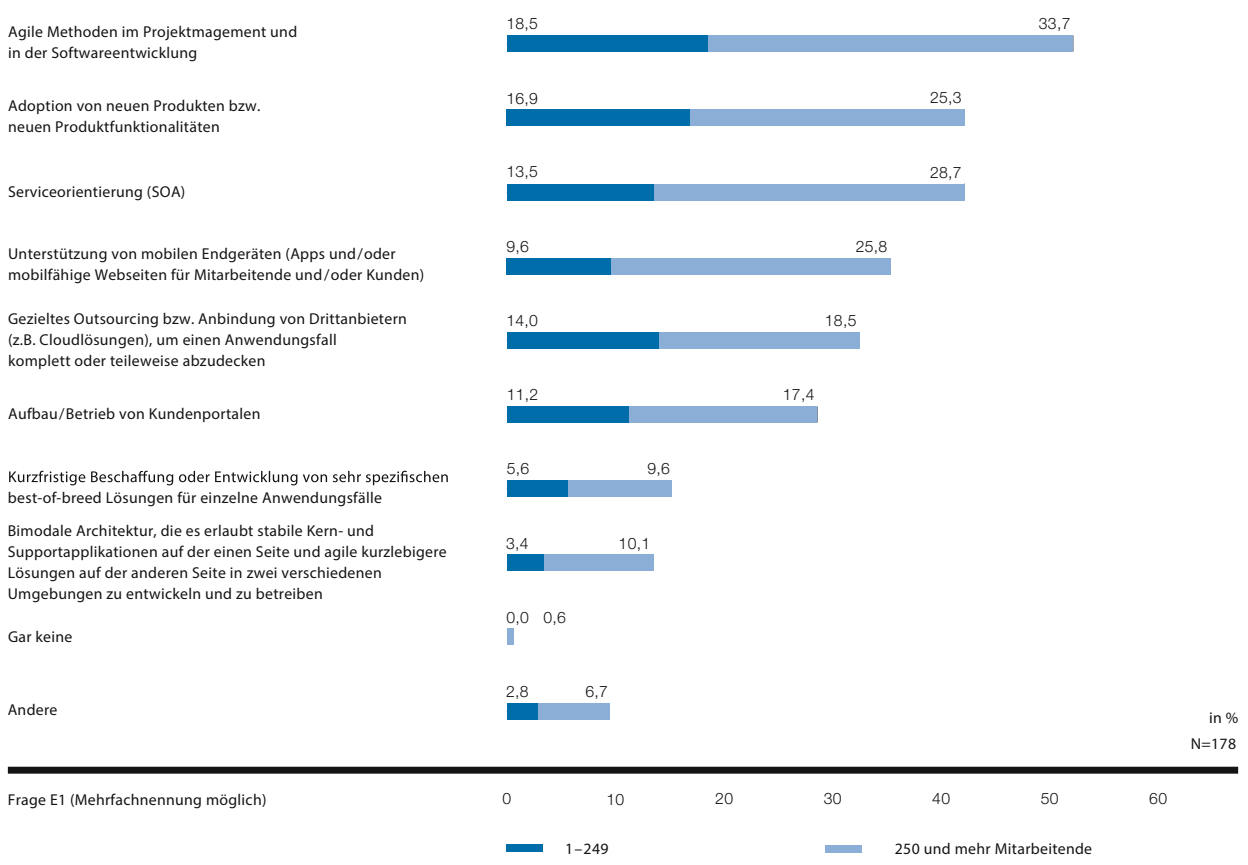

- Abb. 2.14 Reaktionen der IT auf den unternehmerischen und technologischen Wandel

bei der unternehmensweiten Verankerung der Digitalisierung in Form einer Strategie oder Roadmap für die digitale Transformation.

Herkömmliche Methoden und Strukturen der Informatik genügen den dynamischen Anforderungen des digitalen Wandels in Unternehmen häufig nicht. Wie reagieren Unternehmen darauf (- Abb. 2.14)? Für das Projektmanagement und die Softwareentwicklung sind agile Methoden in mehr als der Hälfte der befragten Unternehmen etabliert. Einen ähnlich starken Einfluss auf Kompetenzen und Paradigmen in der Bereitstellung von IT-Leistungen hat Serviceorientierung, die bei immerhin $42 \%$ der befragten Unternehmen als Wegbereiter des digitalen Wandels eingesetzt wird. Die Unterstützung von mobilen Endgeräten ist mit $35 \%$ überraschend niedrig. Sie scheinen für den digitalen Arbeitsplatz oder die Schnittstelle zum Kunden weniger relevant als vermutet (vgl. - Abb. 2.12).

\subsection{Was will der Kunde?}

Die Schaffung positiver Kundenerlebnisse ist ein zentrales Element der digitalen Transformation. Unternehmen investieren in die digitale Erweiterung bestehender Produkte und führen neue Technologien an der Schnittstelle zum Kunden ein. Das alles setzt voraus, dass Unternehmen die vermeintlichen und echten Bedürfnisse ihrer Kunden kennen. Um Kundenbedürfnisse frühzeitiger und unmittelbarer zu erfassen, wurde das Repertoire der klassischen Marktforschung um Methoden wie Prototyping oder Customer Journeys erweitert. Setzen Unternehmen diese Methoden bereits ein? Wie steht es um die Kenntnis der Kundenbedürfnisse und Kundenzufriedenheit in den befragten Unternehmen? 


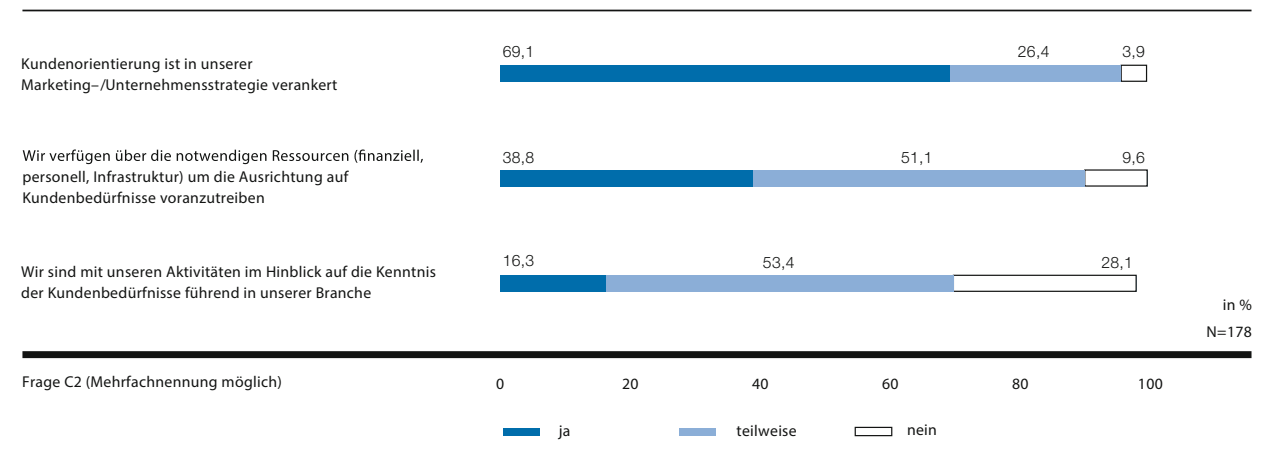

- Abb. 2.15 Ausprägung der Kundenorientierung

Bei nahezu allen befragten Unternehmen (95\%) ist die Kundenorientierung, zumindest auf dem Papier, strategisch verankert (- Abb. 2.15). Ein Blick auf die Umsetzung der Kundenausrichtung zeigt, dass nur $39 \%$ auch vollumfänglich über die notwendigen personellen, finanziellen und technischen Ressourcen hierfür verfügen. Für 51 \% der Unternehmen ist dies zumindest teilweise der Fall. Ob dieser Mitteleinsatz ausreicht, um in Sachen Kundenverständnis und -ausrichtung eine branchenführende Stellung einzunehmen, schätzen die befragten Unternehmen verhalten selbstbewusst ein. Nur rund $16 \%$ sehen sich hier eindeutig in einer Spitzenposition, aber mehr als die Hälfte glauben zumindest punktuell über einen Vorsprung gegenüber den Mitbewerbern zu verfügen.

Methoden, um die Kundenbedürfnisse zu erheben, werden bereits intensiv eingesetzt (- Abb. 2.16). Zum Standard gehört bei den befragten Unternehmen die Auswertung von Kundenreklamationen. Mehr als $70 \%$ tun dies sogar systematisch, weitere $24 \%$ gelegentlich. Auch Kundenbefragungen mittels Fragebogen oder anderer quantitativer Instrumente (79\%) sowie qualitative Kundeninterviews $(75 \%)$ sind regelmässig oder gelegentlich im Einsatz. Neben diesen retrospektiven Massnahmen, die nach dem Kundenerlebnis ansetzen, sind Methoden für eine frühzeitige Erkennung der Kundenbedürfnisse auf dem Vormarsch: Ein Viertel der befragten Unternehmen setzten Prototypen oder Tests mit Kunden vor der Einführung neuer Produkte und Dienstleistungen bereits systematisch ein, um die Akzeptanz neuer Angebote oder Interaktionsformen zu prüfen. Weitere $47 \%$ setzen diese gelegentlich ein. Social Media Monitoring ist bei nahezu zwei Dritteln der befragten Unternehmen im Einsatz, $30 \%$ betreiben es sogar systematisch. Kunden-Communities hingegen sind weniger präsent, werden aber doch bei nahezu der Hälfte der befragten Unternehmen systematisch oder gelegentlich genutzt. Auch Business Intelligence kommt bei der überwiegenden Mehrzahl der Unternehmen zum Einsatz: $71 \%$ erheben regelmässig oder gelegentlich Kennzahlen, die Rückschlüsse auf die Kundenzufriedenheit zulassen und $68 \%$ analysieren Kundendaten. Am unteren Ende der Liste, aber doch von mehr als der Hälfte der befragten Unternehmen systematisch oder gelegentlich angewandt, stehen interaktionsorientierte Erhebungsmethoden, die die Bedürfnisse, Befindlichkeiten und Berührungspunkte der externen Kunden mittels Customer Journey (54\%) und der internen Kunden, sprich der Mitarbeitenden, mittels Employee Journey (40\%) abbilden.

Grosse Unternehmen scheinen die Distanz zum Kunden etwas stärker ausgleichen zu müssen als KMUs. Alle Methoden zur Erhebung der Kundenbedürfnisse werden von grossen Unternehmen ausgeprägter eingesetzt als von KMUs (- Abb. 2.17). Insbesondere beim Einsatz von quantitativen oder stark technologieabhängigen Methoden sind die KMUs deutlich weniger 
Wir analysieren die bei uns eingehenden Beschwerden von Kunden

Wir befragen Kunden mit Hilfe quantitativer Methoden (z.B. mit Fragebogen)

Wir befragen Kunden mit Hilfe qualitativer Methoden (z.B. Interviews, Gruppendiskussionen)

Wir erheben Kennzahlen, die implizit Rückschlüsse auf die Kundenzufriedenheit bzw. den Kundennutzen zulassen ( $z$. B. Verweilzeit auf der Website, Zuwachs Up- und Crossselling, Anzahl Service-Anfragen, Anzahl Reklamationen)

Wir prüfen die Kundenakzeptanz von neuen Angebotskonzepten (Prototypen) direkt mit Kunden (z.B. in Produkttests) bevor wir neue Produkte, Dienstleistungen, Interaktionsformen einführen

Wir sammeln Daten über Einzelkunden und/oder Kundensegmente und werten sie aus

Wir beobachten Kundenmeinungen in Bezug auf unser Unternehmen im World Wide Web (Social Media Monitoring)

Wir nutzen die "Customer Journey», um Bedürfnisse unsere Kunden prozess- und interaktionsorientiert

zu erheben

Wir haben Zugriff auf eine Kunden-Community, die bei kundenspezifischen Fragestellungen/Themen aktiv mit uns zusammenarbeitet

Wir nutzen die «Employee Journey», um die Berührungspunkte der Mitarbeitenden von der Rekrutierung an abzubilden, und die Arbeitsumgebung danach zu gestalten

Andere

Frage C3 (Mehrfachnennung möglich)

\begin{tabular}{|c|c|c|c|}
\hline 71,3 & & 24,2 & 2,2 \\
\hline 39,3 & 39,9 & & 18,5 \\
\hline 30,9 & 44,4 & & 22,5 \\
\hline 34,3 & 37,6 & & 23,0 \\
\hline
\end{tabular}

24,7
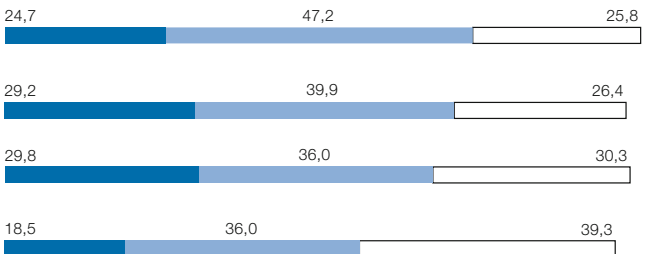

16,9

32,6

44,9

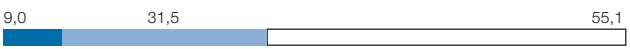

$\begin{array}{llll}0,6 & 0,6 & 3,4 & \text { in } \%\end{array}$

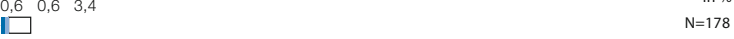

\begin{tabular}{llllll}
\hline 0 & 20 & 40 & 60 & 80 & 100
\end{tabular}

- Abb. 2.16 Methoden für die Erhebung der Bedürfnisse von externen und internen Kunden

Wir analysieren die bei uns eingehenden Beschwerden von Kunden

Wir befragen Kunden mit Hilfe quantitativer Methoden (z.B. mit Fragebogen)

Wir befragen Kunden mit Hilfe qualitativer Methoden (z.B. Interviews, Gruppendiskussionen)

Wir erheben Kennzahlen, die implizit Rückschlüsse auf die Kundenzufriedenheit bzw, den Kundennutzen zulassen (z B Verweilzeit auf der Website, Zuwachs Up- und Cross-selling, Anzahl Service-Anfragen, Anzahl Reklamationen)

Wir prüfen die Kundenakzeptanz von neuen Angebotskonzepten (Prototypen) direkt mit Kunden (z.B. in Produkttests) bevor wir neue Produkte, Dienstleistungen, Interaktionsformen einführen

Wir sammeln Daten über Einzelkunden und/ oder Kundensegmente und werten sie aus

Wir beobachten Kundenmeinungen in Bezug auf unser Unternehmen im Word Wide Web (Social Media Monitoring)

Wir nutzen die "Customer Journey", um Bedürfnisse unserer Kundenprozess- und interaktions-orientiert zu erheben

Wir haben Zugriff auf eine Kunden-Community, die bei kundenspezifischen Fragestellungen Themen aktiv mit uns zusammenarbeitet

Wir nutzen die "Employee Journey", um die Berührungspunkte der Mitarbeitenden von der Rekrutierung a Arbeitsumgebung danach zu gestaten

Andere
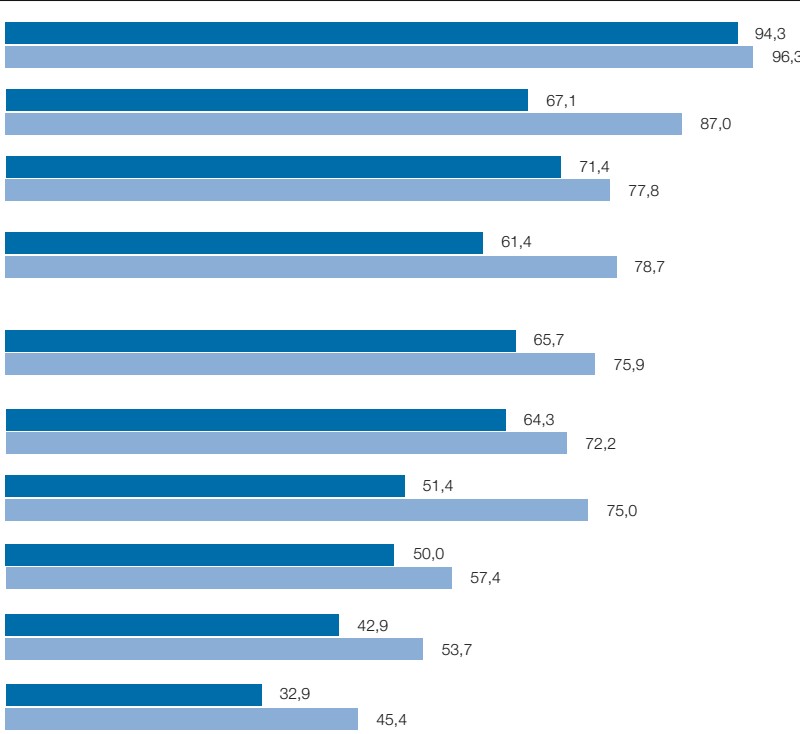

2,9

in \% $\mathrm{N}(\mathrm{KMU})=70$

Frage C3 (Mehrfachnennung möglich)

0

60

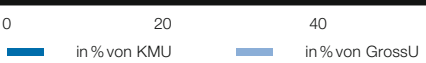

- Abb. 2.17 Methoden für die Erhebung der Bedürfnisse von externen und internen Kunden - «systematisch oder gelegentlich» nach Unternehmensgrösse 
aktiv: $51 \%$ der KMUs versus $75 \%$ der grossen Unternehmen nutzen systematisch oder gelegentlich Social Media Monitoring. Bei quantitativen Umfragen und der Erhebung von Kennzahlen sind die befragten KMUs jeweils um rund $20 \%$ weniger aktiv als die grossen Unternehmen.

\subsection{Kundenperspektive in der Prozessgestaltung und -optimierung}

Unternehmen haben erkannt, dass es wichtig ist, die Bedürfnisse ihrer bestehenden und potenziellen Kunden zu kennen. Dies allein reicht jedoch noch nicht, um den Kundennutzen und die Kundenzufriedenheit zu steigern. Die Erkenntnisse müssen in die strategische Ausrichtung, aber vor allem in die Ausgestaltung von Produkten, Dienstleistungen und des operativen Geschäfts einfliessen. Das Prozessmanagement bietet Ansatzpunkte: Die Visualisierung der Kundenbedürfnisse und -interaktionen in Prozesslandkarten und -modellen könnte wertvolle Einsichten liefern, um Optimierungspotenziale im Front- und Back-End mit Blickrichtung Kundennutzen systematisch zu identifizieren. Werden diese Möglichkeiten und die Synergien zwischen Marketing und Prozessmanagement bereits genutzt? Und in welchen Aktivitäten zur Modernisierung und Optimierung der Kundeninteraktionen schlägt sich dies nieder?

\section{- Visualisierung der Kundenperspektive}

Die aktuelle Befragung bestätigt die Erkenntnis aus den BPM-Studien der letzten Jahre, dass die überwiegende Mehrheit der befragten Unternehmen (87 \%) Prozesse modelliert oder über eine Prozesslandkarte verfügt (- Abb. 2.18). Die angestrebte Transparenz wird also erreicht. Bleibt die Frage, ob diese Transparenz genutzt wird, um den Kundenbedarf oder neuralgische Punkte in der Kundeninteraktion aufzuzeigen. Hier bestätigt sich die Hypothese, dass viele Unternehmen Prozesse ausschliesslich aus der internen Perspektive abbilden (30\%). Auch auf den Prozesslandkarten sind Kundenbedarf oder -beziehung bei weniger als bei einem Drittel der Unternehmen präsent (29\%). In einzelnen Prozessmodellen nutzen 42 \% der Unternehmen die Möglichkeiten, Kundeninteraktionen abzubilden.

Erst $30 \%$ der Unternehmen nutzen die Prozesslandkarte, um die Kundenperspektive zu visualisieren. - Abb. 2.19 zeigt, in welcher Form und Ausprägung Unternehmen dies aktuell umsetzen: $18 \%$ haben ihre Prozesslandkarten bereits auf die Wertschöpfungskette ausgerichtet und visualisieren End-to-End-Prozesse konsequent vom Kundenbedarf bis zur Leistungserfüllung für den Kunden. $11 \%$ visualisieren unterschiedliche Kundengruppen auf ihren Prozess-

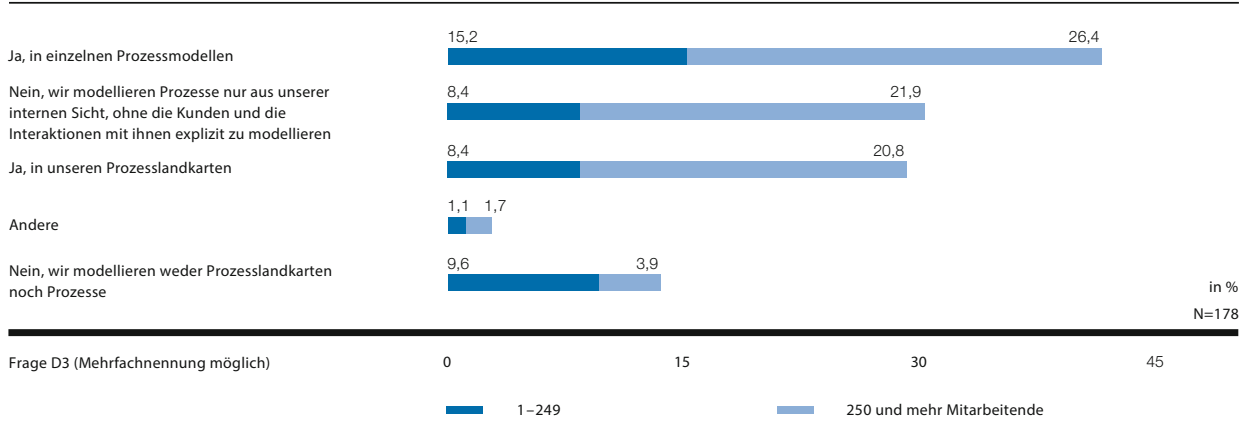

- Abb. 2.18 Visualisierung von Kundeninteraktionen und -bedarf in Prozessmodellen und -landkarten 


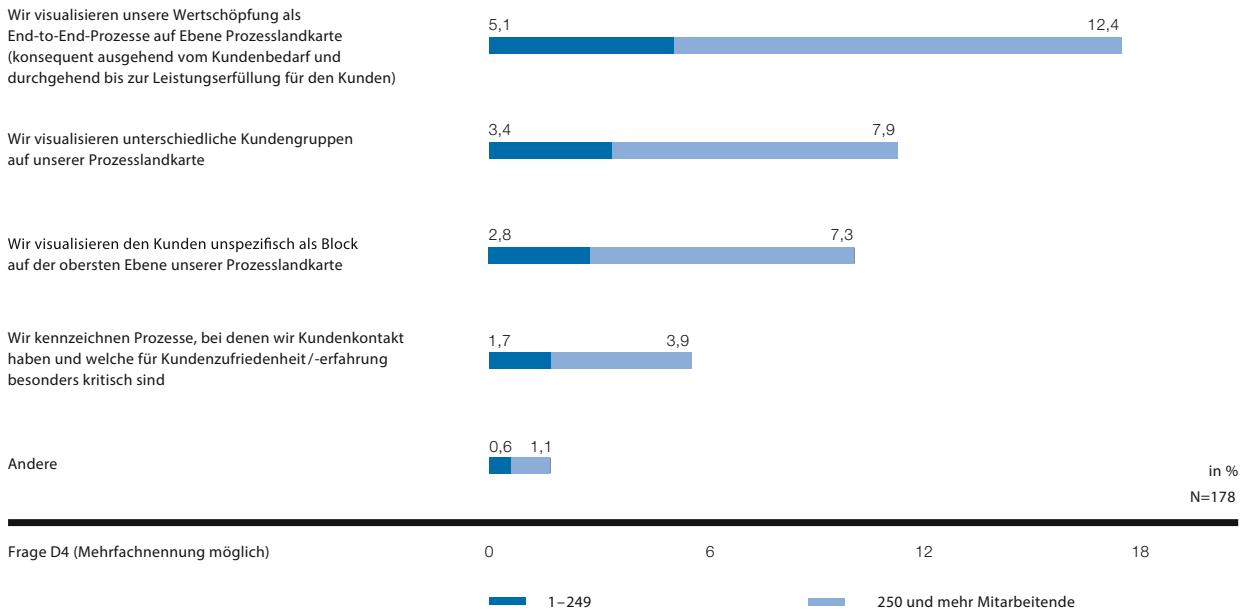

- Abb. 2.19 Visualisierung von Kundeninteraktionen in Prozesslandkarten

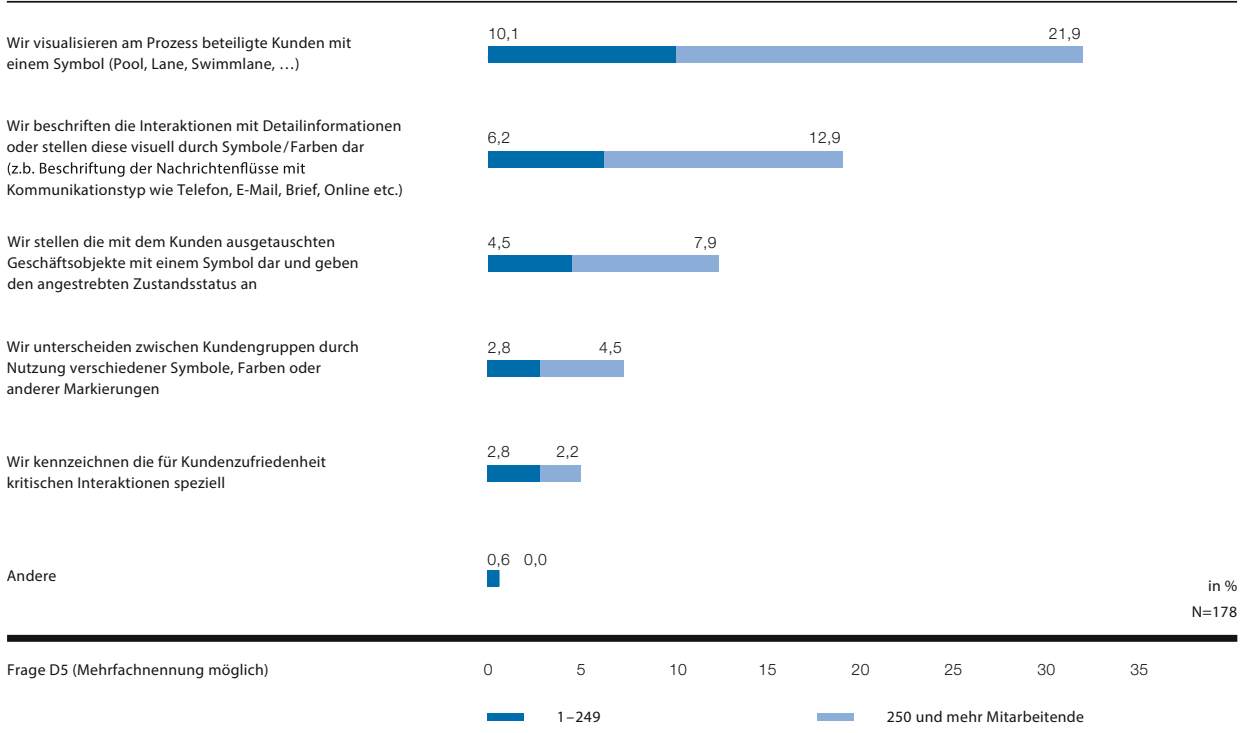

- Abb. 2.20 Visualisierung von Kundeninteraktionen in einzelnen Prozessmodellen

landkarten. Nur knapp $6 \%$ der befragten Unternehmen kennzeichnen ihre kundenkritischen Prozesse, obwohl $28 \%$ systematisch und weitere $57 \%$ gelegentlich die für den Kundennutzen und die Kundenzufriedenheit kritischen Prozesse identifizieren (siehe Abschn. 2.2, - Abb. 2.6). Prozesslandkarten spielen also nur eine geringe Rolle, wenn es darum geht die Erkenntnisse dieser Analysen zu visualisieren. Betrachtet man Prozesslandkarten als zentrales Instrument des strategischen Prozessmanagements, stellt sich die Frage, weshalb sich eine so hochpriorisierte Zielsetzung wie Kundenorientierung kaum in den Prozesslandkarten niederschlägt.

Etwas stärker werden die einzelnen Prozessmodelle genutzt, um die Kundeninteraktionen festzuhalten (32\%, - Abb. 2.20). Dies geschieht primär mit den standardmässig durch Model- 
lierungsnotationen vorgegebenen Darstellungsmöglichkeiten wie Pool, Lane oder Swimmlane bzw. mithilfe von BPMN-Kollaborationsdiagrammen. Erst wenige unterscheiden dabei die verschiedenen Kundengruppen (7\%). Ebenfalls eher selten abgebildet werden die Detailinformationen über den Interaktionskanal (19\%) und die mit den Kunden über den Kanal ausgetauschten Informationen oder Geschäftsobjekte (12\%). Nur $5 \%$ kennzeichnen die neuralgischen Kundeninteraktionen. Prozessverantwortliche und -beteiligte erhalten also kaum Orientierungshilfen aus Prozessmodellen, wenn sie Prozesse kundenzentrisch betrachten oder optimieren wollen.

\section{- Customer Journeys für die Prozessoptimierung}

Bei der Frage nach den genutzten Methoden, um Kundenbedürfnisse zu erheben, gaben $54 \%$ an, dass sie Customer Journey nutzen um Bedürfnisse, Befindlichkeiten und Interaktionen ihrer Kunden zu erheben. Fast $20 \%$ tun dies systematisch (vgl. - Abb. 2.16). Mehr als die Hälfte der Organisationen, die die Customer Journey bereits einsetzen, nutzt die Erkenntnisse für die Gestaltung oder Optimierung kundenorientierter Prozesse (vgl. - Abb. 2.21). Die Customer Journey wird also nicht nur als modernes Instrument in Marketinghänden betrachtet, sondern in den Organisationen zielgerichtet für die Optimierung der Prozesse eingesetzt. Das Potenzial, Prozessdesign und Customer Journey Mapping zu verbinden, ist erkannt, wird aber sowohl von grossen Unternehmen als auch von KMUs noch nicht vollumfänglich ausgeschöpft.

Bei der Employee Journey zeigt sich ein ähnliches Bild (• Abb.2.22). Der Einsatz für die Prozessoptimierung ist jedoch etwas schwächer ausgeprägt und erwartungsgemäss stärker durch Grossunternehmen geprägt. Die Methode der Employee Journey, das heisst, die Adaption der Customer Journey auf die Bedürfnisse und Interaktionen mit Mitarbeitenden, ist bei der Mehrzahl der befragten Unternehmen noch nicht im Einsatz (siehe - Abb. 2.16, 60 \%). Dass weniger als die Hälfte der verbleibenden 40 \% der Unternehmen, die die Employee Journey systematisch

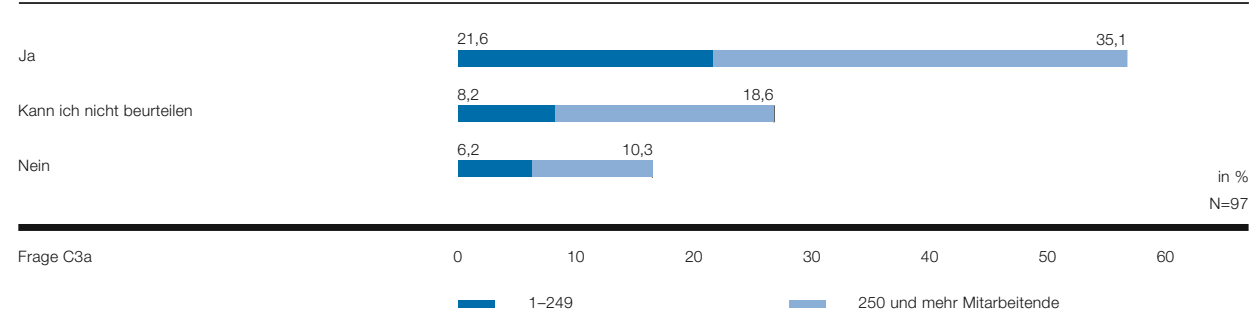

- Abb. 2.21 Nutzung der «Customer Journey«-Erkenntnisse für die kundenorientierte Prozessgestaltung/-optimierung

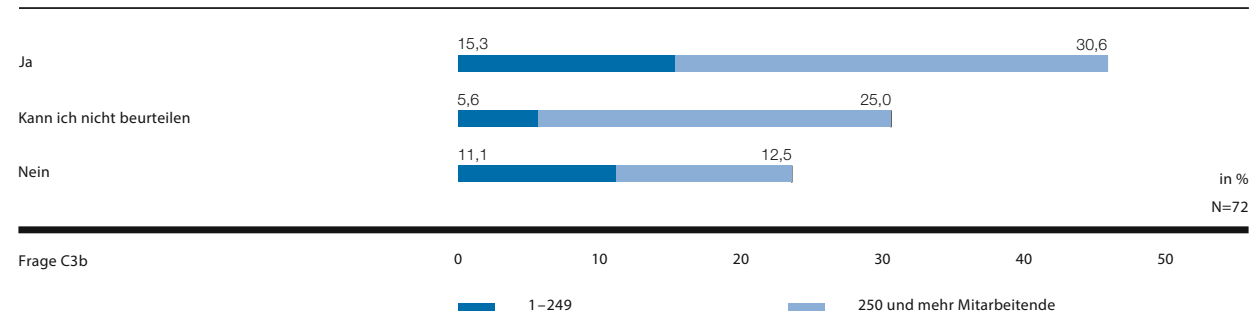

- Abb. 2.22 Nutzung der «Employee Journey«-Erkenntnisse für mitarbeiterorientierte Prozessgestaltung/-optimierung 
oder gelegentlich einsetzen, die Erkenntnisse nur begrenzt für die Prozessgestaltung einsetzen, könnte den Schluss nahelegen, dass der Fokus bei der Prozessoptimierungen mit internem Fokus traditionell auf Harmonisierung und Standardisierung liegt und nicht auf Mitarbeiterbefähigung und -bindung. Vor dem Hintergrund allerdings, dass mehr als $80 \%$ der befragten Unternehmen die Einführung innovativer digitaler Arbeitsplätze für ihre Mitarbeitenden als Aktionsfeld der digitalen Transformation nennen (siehe Abschn. 2.3, - Abb. 2.12), scheint schlichtweg die Employee Journey als Methode in diesem Kontext noch zu wenig bekannt oder - insbesondere in KMUs - zu wenig relevant zu sein.

\section{- Gestaltung der Kundeninteraktion}

Kunden neue Interaktionsmöglichkeiten mit dem Unternehmen und dessen Produkten und Dienstleistungen zu eröffnen, ist ein wesentlicher Aspekt der digitalen Transformation. Die oben beschriebene konzeptionelle Sicht auf die Kundenperspektive in Form von Customer Journeys oder Prozessmodellen bildet nur die Grundlage für die Ausgestaltung der Kundenbeziehung. Doch welche Massnahmen ergreifen Unternehmen, um das Kundenerlebnis im operativen Geschäft effektiv zu verändern (- Abb. 2.23)?

Mehr als drei Viertel der befragten Unternehmen erweitern oder optimieren digitale Touchpoints oder Kanäle, allerdings überwiegend taktisch/punktuell (52\%) und weniger strategisch/systematisch (26\%). Dies deckt sich mit dem medial geprägten Bild, dass Unternehmen digitale Transformation typischerweise in Form von Einzelinitiativen betreiben, indem sie beispielsweise eine mobile App lancieren oder eine Website modernisieren. Die gewünschte Durchgängigkeit der physischen und digitalen Kundenerlebnisse scheinen Unternehmen dabei nicht aus den Augen zu verlieren, wobei auch diese eher punktuell (43\%) als systematisch (22\%) stattfindet. Die physischen Kanäle werden im Zeitalter des technologischen Wandels

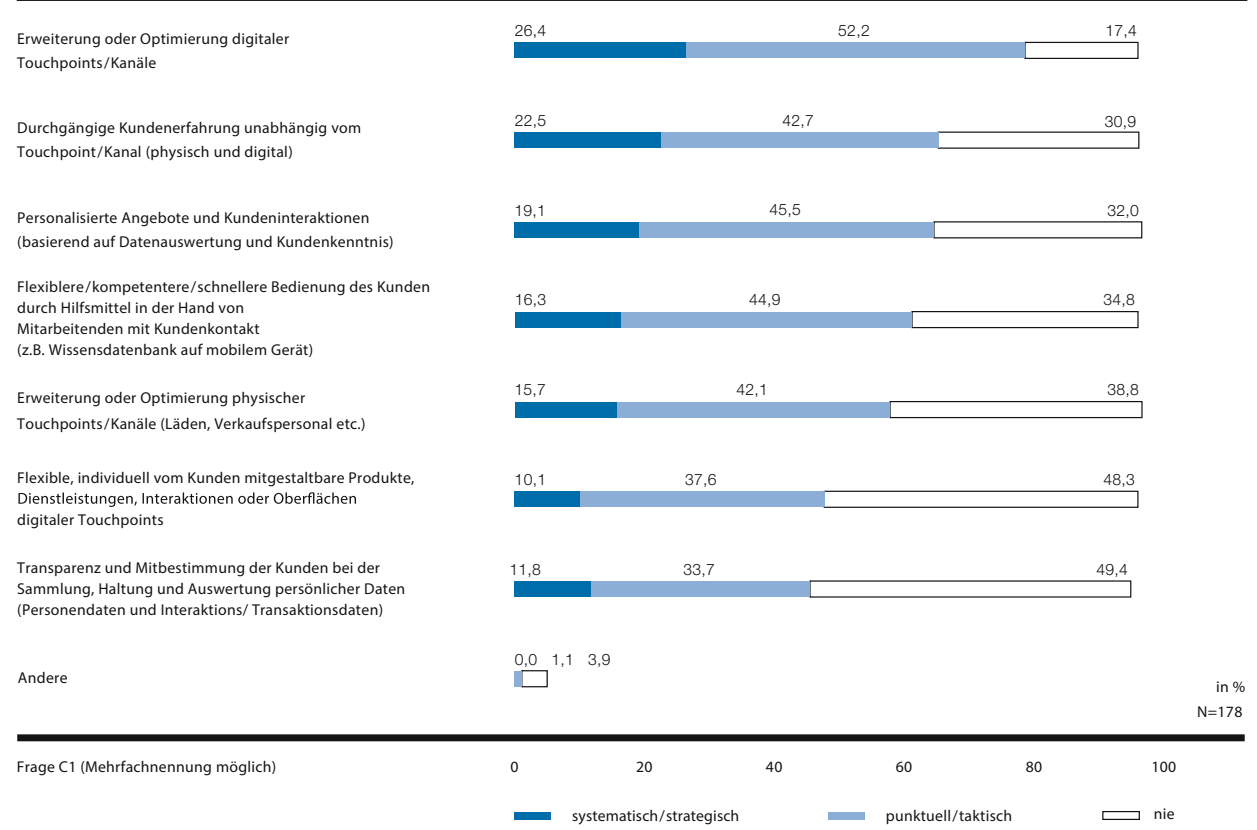

- Abb.2.23 Aktivitäten zur Gestaltung der Kundeninteraktion 
nicht vernachlässigt. Die Mehrzahl der befragten Unternehmen (58\%) investiert weiterhin systematisch oder punktuell in die Optimierung ihrer physischen Kanäle wie beispielsweise Ladenflächen. Ähnlich relevant ist in diesem Zusammenhang die Befähigung der Mitarbeitenden mit Kundenkontakt durch digitale Hilfsmittel (61\%). Bemerkenswert ist, dass die Erweiterung der digitalen Kanäle immerhin bei bereits fast der Hälfte der befragten Unternehmen so weit geht, die Kunden ihre Oberflächen, Interaktionen oder gar Produkte und Dienstleistungen selbst gestalten zu lassen (48\%). Allerdings bieten aktuell nur erst $10 \%$ der befragten Unternehmen diese fortgeschrittene Form der Digitalisierung ihren Kunden systematisch an. Interessant ist, dass obwohl die Sicherstellung von Datenschutz und -sicherheit im Kontext der Digitalisierung bei rund der Hälfte aller befragten Unternehmen bereits realisiert (27\%) oder in der Umsetzung ist (25\%; vgl. Abschn. 2.3, - Abb. 2.12), die Transparenz der Datenhaltung für die Kunden noch kaum ein strategisches Thema auf Unternehmensebene ist (12\%). Wenn überhaupt wird das Bedürfnis der Kunden, zu wissen oder gar zu bestimmen, welche Daten wo und wie lange gespeichert werden und zu welchem Zweck Daten verwendet werden, nur punktuell berücksichtigt (34\%). Dies legt den Schluss nahe, dass sich viele Unternehmen darauf beschränken, die gesetzlichen Anforderungen in Sachen Datenschutz zu erfüllen, aber über diese Pflichtübung hinaus keine weiteren Anstrengungen unternehmen, ihren Kunden mehr Kontrolle über die gespeicherten Daten zu gewähren.

Grosse Unternehmen gehen die Optimierung der Kundeninteraktionen etwas aktiver an, insbesondere wenn es um die Touchpoints, sowohl die digitalen als auch die physischen, aber auch um die Durchgängigkeit der verschiedenen Kanäle geht (• Abb. 2.24). Dafür gehen KMUs vergleichsweise stärker auf den einzelnen Kunden ein und lassen ihn Produkte, Dienstleistungen, Interaktionen oder Oberflächen digitaler Touchpoints individuell und flexibel mitgestalten (59\% der befragten KMUs versus $41 \%$ der grossen Unternehmen).

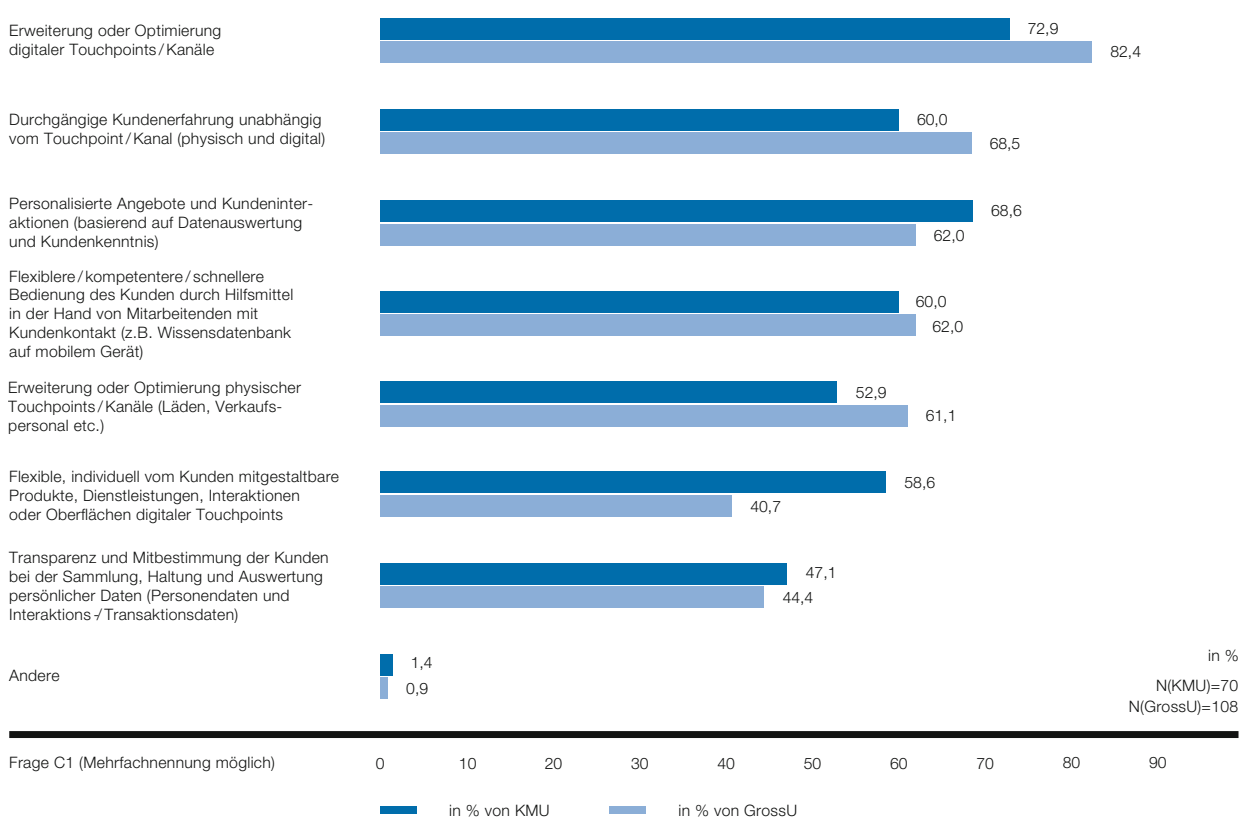

- Abb. 2.24 Aktivitäten zur Gestaltung der Kundeninteraktion - «systematisch oder punktuell» nach Unternehmensgrösse 


\subsection{Kundendaten: Quellen, Nutzung und Einsatz für die Prozessausführung}

Die Studie geht von der Hypothese aus, dass Kundendaten und operative Prozessdaten der Treibstoff der digitalen Transformation sein können. Sie spielen eine zentrale Rolle, wenn es um die kundenorientierte Ausgestaltung, Flexibilisierung und Optimierung der Prozesse geht. Doch nutzen Unternehmen dieses Potenzial? Aus welchen Quellen und zu welchen Zwecken beziehen sie ihr Kundenwissen und nutzen sie es für die Gestaltung des Kundenerlebnisses und der Prozessausführung? Nehmen Unternehmen dabei die Verantwortung, die Datenhaltung für ihre Kunden transparent zu gestalten, wahr?

Die etablierten Marketinginstrumente Kundenumfrage (77\%) und dedizierte Kundendatenbanken oder CRM-Systeme (67\%) sind für die befragten Unternehmen die wichtigsten Quellen für Kundendaten (• Abb. 2.25). Aber auch Soziale Medien sind mit $57 \%$ auf dem Vormarsch, wenn auch nur begrenzt systematisch ausgewertet (15\%). Die Auswertung operativer Daten aus Kern- und Transaktionssystemen spielt durchaus eine grosse Rolle (52\%), aber es bleibt noch Potenzial, diese Quellen systematisch für die Analyse des Kundenverhaltens zu nutzen. Kaum auf dem Radar hingegen sind IoT-Technologien, die abhängig von Branche und Anwendungsfeld sicher eingesetzt oder getestet werden, aber deren Einsatzfeld offensichtlich (noch) nicht in der Analyse des Kundenverhaltens gesehen wird.

KMUs und grosse Unternehmen nutzen Kundendatenbanken oder CRM-Systeme und Kundenumfragen in etwa in gleichem Masse, bei allen anderen Quellen sind die grossen Unternehmen aktiver (- Abb. 2.26). Am deutlichsten ist der Vorsprung der Grossen bei der Nutzung der Daten aus Kern- und Transaktionssystemen. Fast zwei Drittel der befragten grossen Unternehmen nutzt sie, aber nur ein Drittel der KMUs.

Um Kundendaten zentral für alle Unternehmensfunktionen und -systeme bereitzuhalten, haben sich Kundendatenbanken oder funktionsreichere CRM-Systeme etabliert (- Abb. 2.27). $59 \%$ der befragten Unternehmen verfügen über diese zentralen Kundeninformationssysteme, aber nur $41 \%$ nutzen sie für Analysen und Berichte. Eine Ursache könnte im Informationsgehalt der Systeme liegen: Nur 38 \% der Unternehmen speichern in ihren CRM-Systemen über die reinen Kundenstammdaten hinaus auch Informationen zu Kundentransaktionen und -interaktionen. Ähnlich schwach ausgeprägt ist auch der Informationsfluss von den CRM-Sys-

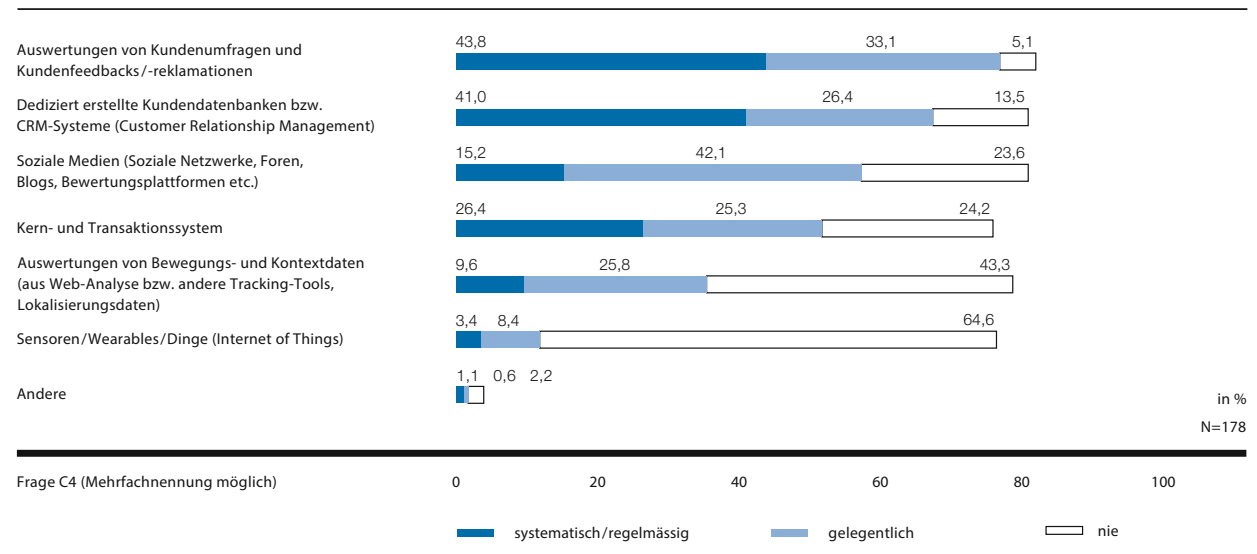

- Abb.2.25 Quelle und Regelmässigkeit der Erhebung von Kundendaten 


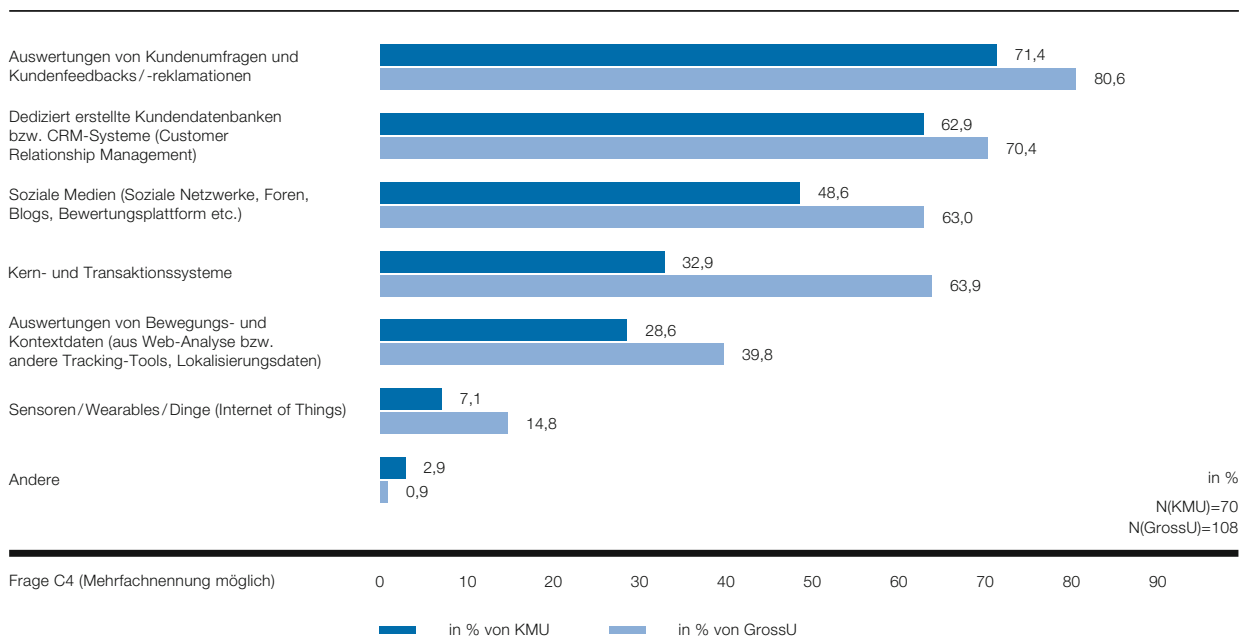

- Abb. 2.26 Quelle und Regelmässigkeit der Erhebung von Kundendaten - nach Unternehmensgrösse

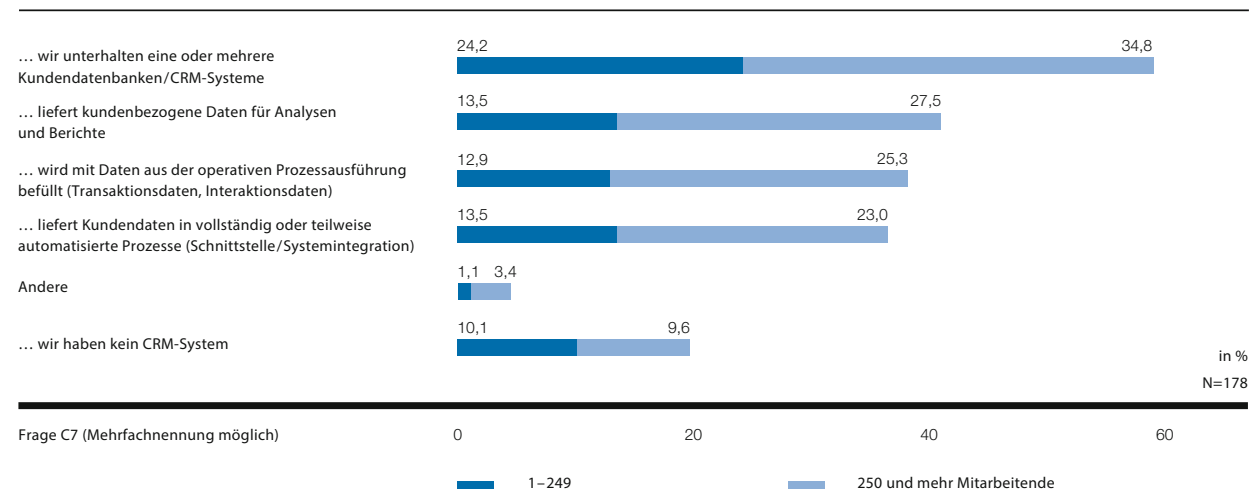

- Abb. 2.27 Verbreitung und Einsatzweise von CRM-Systemen

temen in IT-Systeme, die die Prozessausführung automatisieren (36\%). Beide Erkenntnisse führen zum Schluss, dass CRM-Systeme noch unzureichend integriert sind. Eine mögliche Grundlage für die kundenorientierte Prozessgestaltung und -steuerung scheint somit noch nicht ausreichend geschaffen beziehungsweise ausgeschöpft.

Kundendaten nutzen die befragten Unternehmen in erster Linie kumuliert und asynchron, das heisst, Daten über Kunden und deren Verhalten werden zunächst gesammelt und ausgewertet, um dann Rückschlüsse zu ziehen und Massnahmen abzuleiten (- Abb. 2.28). Diese Erkenntnisse werden in erster Line verwendet, um Produkte und Dienstleistungen zu entwickeln oder anzupassen (54\%). KMUs sind in diesem Punkt führend (• Abb. 2.29). (61 \% der befragten KMUs versus $49 \%$ der grossen Unternehmen.) Kundendaten werden ausserdem ausgewertet, um Webseiten oder Ladenflächen (48 \%) zu optimieren und um Muster und Tendenzen im Kundenbedarf zu erkennen sowie individuelle Empfehlungen ableiten zu können (44\%). Für das Prozessdesign und die kundenorientierte Optimierung von Prozessen werden diese Kundendatenauswertungen hingegen weniger genutzt (35\%). Synchrone Reaktionen auf 
... um neue Produkte und Dienstleistungen zu entwickeln oder bestehende anzupassen $\ldots$ um das Kundenerlebnis für bestehende Kunden zu
verbessern (z.B. Optimierung Website, Ladengestaltung)

.. um Tendenzen, Muster und Entwicklungen zu erkennen und diese für individuelle Empfehlungen oder Reaktionen zu nutzen (predictive analytics)

. für das Prozessdesign, um Prozesse mit Blick auf Kundenorientierung zu gestalten und zu optimieren

.. für die Prozessausführung, um einen Prozessverlauf in Echtzeit kunden-/kontextspezifisch anpassen zu können

Andere

$$
1,1 \quad 0,0
$$
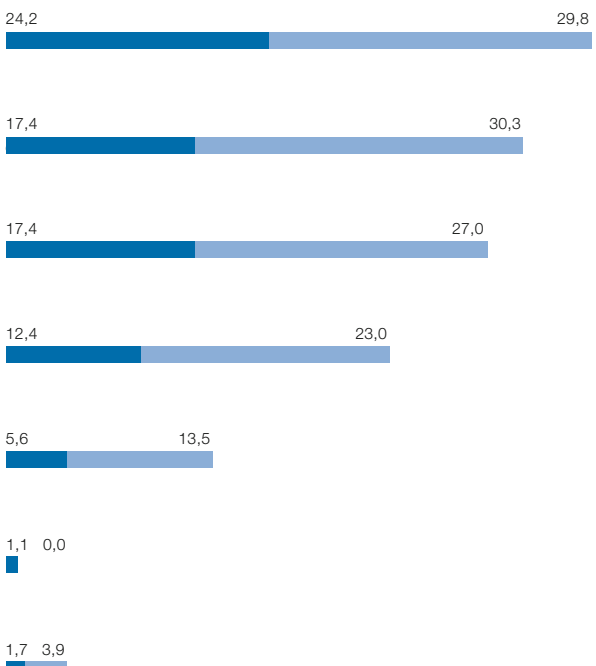

$\begin{array}{ll}1,7 & 3,9\end{array}$

Wir nutzen unsere Kundendaten nicht

in \% $\mathrm{N}=178$

- Abb. 2.28 Nutzung von Kundendaten

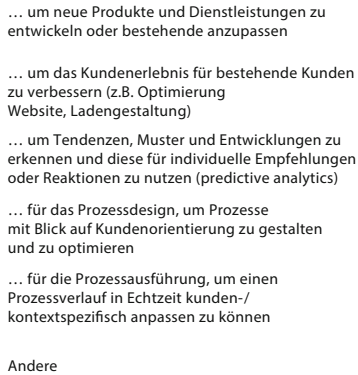

.. um Tendenzen, Muster und Entwicklungen zu erkennen und diese für individuelle Empfehlungen oder Reaktionen zu nutzen (predictive analytics)

.. für das Prozessdesign, um Prozesse mit Blick auf Kundenorientierung zu gestalten und zu optimieren

.. für die Prozessausführung, um einen Prozessverlauf in Echtzeit kunden- $/$ kontextspezifisch anpassen zu können

Andere

Wir nutzen unsere Kundendaten nicht
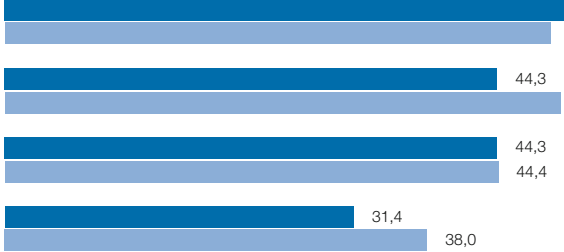

14,3

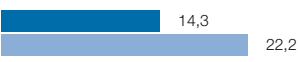

2,9

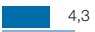

0

10

20

30

40

50

60

70

- Abb.2.29 Nutzung von Kundendaten - nach Unternehmensgrösse

das Verhalten eines individuellen Kunden, die es erlauben, den Prozess in Echtzeit situationsspezifisch anzupassen, sind erst knapp bei einem Fünftel der befragten Unternehmen Realität (19\%). Es besteht also noch ungenutztes Potenzial, Kundendaten systematischer für flexiblere Prozesse und bessere Kundenerlebnisse einzusetzen. 


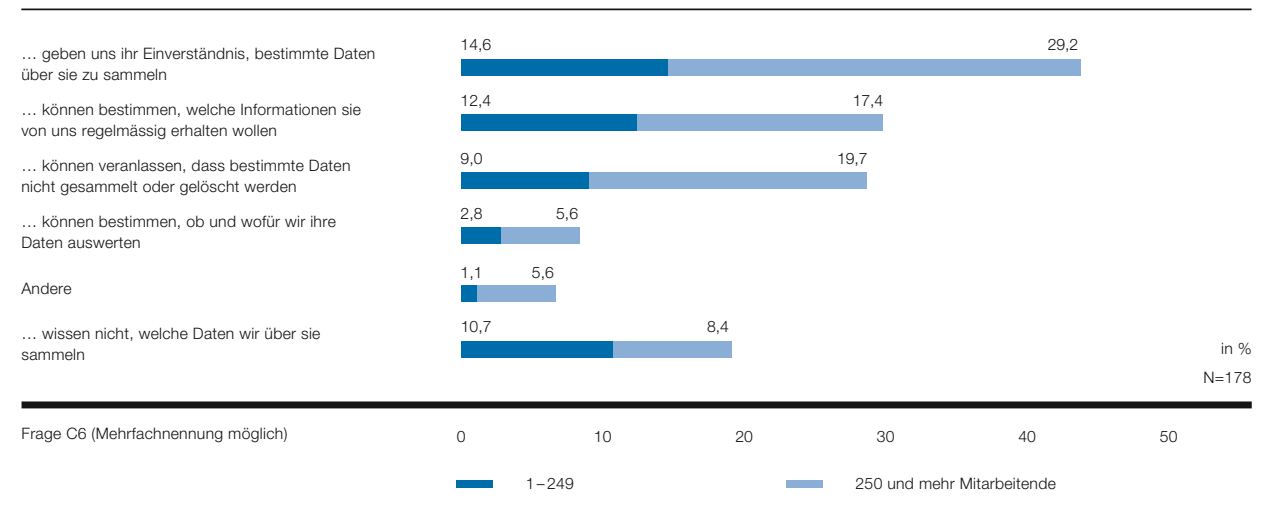

D Abb. 2.30 Einflussmöglichkeiten der Kunden auf die Datenhaltung (»Unsere Kunden ...«)

\section{- Beschränkte Datenherrschaft}

Es bestätigt sich der Eindruck (siehe Abb. 2.23, Abschn. 2.5), dass die befragten Unternehmen noch kaum die Notwendigkeit sehen, ihren Kunden über das gesetzliche Mindestmass hinaus Kontrolle oder zumindest Transparenz über die gespeicherten Kundendaten zu gewähren (- Abb. 2.30). Weniger als die Hälfte der Unternehmen geben an, zumindest das Einverständnis der Kunden zur Datensammlung einzuholen (44\%). Die Notwendigkeit, dies zu tun, ist unter Umständen kontextabhängig wie auch die Möglichkeit, dass Kunden die Speicherung ihrer Daten unterbinden oder deren Löschung verlangen können (29\%). So kann es in bestimmten Branchen und Anwendungsbereichen regulatorische Rahmenbedingungen geben, die es explizit erforderlich machen, Daten oder Kundenkommunikationen aufzubewahren das heisst nicht zu löschen. Insgesamt scheint das Bedürfnis der Kunden nach Transparenz bei den befragten Unternehmen noch nicht spürbar beziehungsweise noch nicht adressiert zu sein: $19 \%$ geben an, dass ihre Kunden nicht wissen, welche Daten das Unternehmen über sie sammelt und nur $8 \%$ der Unternehmen räumen ihren Kunden ein Mitbestimmungsrecht darüber ein, ob und wofür Kundendaten gesammelt werden. Die Themen Transparenz der Datenhaltung und persönliche Datenherrschaft scheinen also noch nicht ausreichend adressiert zu sein.

Open Access Dieses Kapitel wird unter der Creative Commons Namensnennung 4.0 International Lizenz (http://creativecommons.org/licenses/by/4.0/deed.de) veröffentlicht, welche die Nutzung, Vervielfältigung, Bearbeitung, Verbreitung und Wiedergabe in jeglichem Medium und Format erlaubt, sofern Sie den/die ursprünglichen Autor(en) und die Quelle ordnungsgemäß nennen, einen Link zur Creative Commons Lizenz beifügen und angeben, ob Änderungen vorgenommen wurden.

Die in diesem Kapitel enthaltenen Bilder und sonstiges Drittmaterial unterliegen ebenfalls der genannten Creative Commons Lizenz, sofern sich aus der Abbildungslegende nichts anderes ergibt. Sofern das betreffende Material nicht unter der genannten Creative Commons Lizenz steht und die betreffende Handlung nicht nach gesetzlichen Vorschriften erlaubt ist, ist für die oben aufgeführten Weiterverwendungen des Materials die Einwilligung des jeweiligen Rechteinhabers einzuholen.

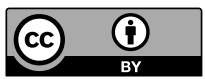

